Check for updates

Cite this: RSC Adv., 2019, 9, 39147

\title{
Synthesis and anion recognition studies of new oligomethylene bis(nitrophenylureylbenzamide) receptors $\uparrow$
}

\author{
José García-Elías, ${ }^{\text {ab }}$ Adrián Ochoa-Terán, (D) *a Anatoli K. Yatsimirsky, ${ }^{c}$ \\ Hisila Santacruz Ortega, ${ }^{d}$ Karen Ochoa-Lara, D ${ }^{* d}$ Luis Miguel López-Martínez, ${ }^{2}$ \\ Christian L. Castro-Riquelme, ${ }^{a}$ Ángel L. García, ${ }^{a}$ Domingo Madrigal-Peralta, ${ }^{a}$ \\ Victoria Labastida-Galván ${ }^{\mathrm{e}}$ and Mario Ordoñez ${ }^{\mathrm{e}}$
}

\begin{abstract}
A new series of oligomethylene bis(nitrophenylureylbenzamide) receptors were synthesized varying the relative position of the urea and amide groups (ortho 4 and meta 8 ) and the length of the oligomethylene chain $\left(\mathrm{C}_{2}\right.$ to $\left.\mathrm{C}_{8}\right)$. An anion recognition study was performed with TBAX salts $\left(X=A c O^{-}\right.$, $\mathrm{BzO}^{-}, \mathrm{F}^{-}, \mathrm{H}_{2} \mathrm{PO}_{4}^{-}$, and $\mathrm{HP}_{2} \mathrm{O}_{7}{ }^{3-}$ ) by $\mathrm{UV}$-vis and ${ }^{1} \mathrm{H} \mathrm{NMR}$. The flexibility of these receptors allows a cooperative effect of both ureylbenzamide units in the receptors. Noteworthy, the ortho position favored the $1: 1$ stoichiometry in the complexes with the carboxylates. The formation of $2: 1$ receptoranion complexes with both types of receptors 4 and 8 and with hydrogen pyrophosphate and high log $K$ values obtained were very significant in this work. The NMR studies evidenced the formation of supramolecular complexes, even in a competitive solvent, such as DMSO.
\end{abstract}

Received 25th July 2019

Accepted 12th November 2019

DOI: $10.1039 / \mathrm{c} 9 \mathrm{ra05783h}$

rsc.li/rsc-advances

\section{Introduction}

Artificial receptors capable of interacting with anions is a very important subject in supramolecular chemistry because of their participation in biological processes, like function, detection, recognition, catalysis, and transport through the cellular membrane, among others. ${ }^{1-3}$ There are many reports related to anion recognition by urea-based organic receptors. The urea group is a good hydrogen bond donor due to the presence of $\mathrm{N}-\mathrm{H}$ bonds, and due to their functionality and directionality. ${ }^{4,5}$ These characteristics play a very important role in the selective recognition of anions with different geometries. The selectivity is related to the energy of the receptor-anion interaction. The strongest hydrogen bond interactions are established with the most basic of anions, such as fluoride, carboxylates, and inorganic oxoanions. From a recent point of view, all the hydrogen bonds behave as proton-transfer reactions. ${ }^{6}$ Therefore, for

${ }^{a}$ Centro de Graduados e Investigación en Química, Tecnológico Nacional de México/IT de Tijuana, Tijuana, México. E-mail: ochoa@tectijuana.mx

${ }^{b}$ Unidad Académica de Ciencias de la Tierra, Universidad Autónoma de Zacatecas, Zacatecas, México

'Facultad de Química, Universidad Nacional Autónoma de México, Ciudad de México, México

${ }^{d}$ Departamento de Investigación en Polímeros y Materiales, Universidad de Sonora, Hermosillo, Sonora, México.E-mail: karenol@polimeros.unison.mx

${ }^{e}$ Centro de Investigaciones Químicas-(IICBA), Universidad Autónoma del Estado de Morelos, Cuernavaca, Mexico

$\dagger$ Electronic supplementary information (ESI) available. See DOI: 10.1039/c9ra05783h a receptor that contains an acid $\mathrm{N}-\mathrm{H}$, the affinity is mainly related to the anion basicity or the $\mathrm{p} K \alpha$ of its conjugated acid. ${ }^{7}$

In this context, nitrophenylureas have been prepared due to the major acidity caused by the resonance effect of the nitro group. ${ }^{8}$ Also, this group allows the study of anion recognition study by spectrophotometric changes; whereby the absorption bands shift to higher wavelengths in complexes with anions, or a new band appears when an acid-base reaction occurs. ${ }^{5}$ The effect in the analytical response and of the interaction with anions of nitrophenylurea receptors when varying the position of the nitro group has been studied, and it was found that the position of the nitro group influences the acidity of the urea hydrogens and the relative affinity toward different anions. ${ }^{6}$

Gunnlaugsson and coworkers ${ }^{9}$ prepared receptors with urea and acetamide groups by changing the relative position of both groups (ortho, meta, and para). The acetamide position had a clear effect over the selectivity and stoichiometry in the complexes with anions. The interaction occurred only in the urea group with one equivalent of the anion in the meta and para receptors, while the interaction with the amide hydrogen occurred forming 1:2 receptor:anion complexes. They proposed this behavior as a "positive allosteric effect" promoted by conformational changes. Interestingly, the ortho receptor formed only $1: 1$ complexes and the ${ }^{1} \mathrm{H}$ NMR spectra showed the participation of the three hydrogens, which indicated a cooperative interaction of the amide hydrogen with the anions.

Bis(urea) receptors are excellent hosts for anions with diverse geometries due to the high number of $\mathrm{N}-\mathrm{H}$ bonds in their 
structure. In addition, these receptors display high preorganization and cooperativity, which enhance the complexation process, ${ }^{\mathbf{1 0}}$ and exhibit a limited number of stable configurations for the interaction with the anion. ${ }^{\mathbf{1 1 , 1 2}}$ Each group forms two hydrogen bonds with the anion, the strength of which may differ depending on the ligand nature, the distance between each urea group, and the size and geometry of the anion..$^{\mathbf{8 1 3 , 1 4}}$

Several bis(urea) receptors for the molecular recognition of anions are reported in the literature. Chauhan and coworkers ${ }^{13}$ prepared four azo bis(urea/tiourea) receptors bearing phenyl or nitrophenyl substituents in the urea group for the colorimetric detection of anions. The affinity of these receptors was in the order $\mathrm{F}^{-}>\mathrm{AcO}^{-}>\mathrm{H}_{2} \mathrm{PO}_{4}{ }^{-}$with a change of color from yellow to red. The ${ }^{1} \mathrm{H}$ NMR studies demonstrated that the hydrogen bond in the urea group induced different effects in the aromatic signals, whereby the enhancement of the electronic density in the phenyl ring generated a shift of the signals to upfield due to the electronic enrichment, while the polarization of the $\mathrm{C}-\mathrm{H}$ bonds through the space caused a deprotecting effect and shifted the signals to downfield.

The most studied bis(urea) receptors are the $N, N^{\prime}-1,3-$ phenylenbis $\left(N^{\prime}\right.$-urea/tiourea) derivatives, initially studied by Nishizawa. ${ }^{14}$ Complexation studies by ${ }^{1} \mathrm{H}$ NMR in DMSO revealed association constant values of $110 \mathrm{M}^{-1}$ and $43 \mathrm{M}^{-1}$ with dihydrogen phosphate and acetate, respectively. ${ }^{15}$ Then, these receptors showed high affinity toward anions by electrochemical methods. ${ }^{16}$ The complex stoichiometry with acetate was found to be $1: 1$ by UV-vis measurements and the reported association constant was $210 \mathrm{M}^{-1} \cdot{ }^{17}$ A colorimetric anion sensor was developed with a bis(urea) receptor synthetized from 4,5dimethyl-1,2-phenylenediamine and nitrophenyl isocyanate. ${ }^{\mathbf{1 8}}$ Spectroscopic changes were observed only with fluoride and acetate anions, indicating selectivity over the other studied anions.

Caltagirone and coworkers ${ }^{19}$ synthesized a series of six bis(urea) receptors using the diamines 1,3-bis(aminomethyl) benzene and 2,6-bis(aminomethyl)pyridine, and the isocianates phenyl : 2-nitrophenyl and 1-naphthyl isocyanates. The binding properties of these receptors were studied in DMSO with acetate, benzoate, glutarate, malonate, dihydrogen phosphate hydrogenpyrophosphate, triphosphate, AMP, and ADP by UV-vis spectroscopy and ${ }^{1} \mathrm{H}$ NMR. These receptors showed high affinity toward acetate, benzoate, and dihydrogen phosphate. The values of the association constants were in the range of 88 to $299 \mathrm{M}^{-1}$ for acetate, 65 to $109 \mathrm{M}^{-1}$ for benzoate, and 167 to 698 $\mathbf{M}^{-1}$ for dihydrogenphosphate. Noteworthy, the hydrogen pyrophosphate caused a partial or complete deprotonation to most of the receptors, allowing the colorimetric detection of this anion in DMSO. The association constants evaluated for two of these receptors were 5840 and $>10^{4} \mathrm{M}^{-1}$, respectively.

All the receptors previously described have a rigid connection between the two urea units, mainly alkenyl or aromatic backbones, which confers some structural preorganization in the anion recognition. Continuing with our interest in the synthesis of new receptors and the study of anion recognition, ${ }^{20}$ a series of new oligomethylene bis(4-nitrophenylureylbenzamide) receptors bearing the urea and amide groups in the ortho or meta position, were synthesized and evaluated as anion receptors. We envisioned that these bifunctional receptors with flexible oligomethylene chains may be modulated in their acidity, with the cooperativity of the binding groups and the size of the receptors cavities allowing interaction with anions of different sizes and geometries (Fig. 1).

\section{Experimental}

\section{Materials and equipment}

All chemicals used in the study were obtained from commercial suppliers and used without further purification. ${ }^{1} \mathrm{H}$ NMR spectra were recorded using a Bruker $400 \mathrm{MHz}$ NMR spectrometer at a probe temperature of $30{ }^{\circ} \mathrm{C}$ with TMS as the internal standard. Electronic impact mass spectra were obtained by direct insertion in an Agilent 5975C mass spectrometer; and the intensities are reported as a relative percentage to the base peak corresponding to an $m / z$ value. FAB mass spectra and HRMS were recorded in an MStation JMS-700 JEOL. UV-vis absorption spectra were obtained using a Cary 300 spectrophotometer.

\section{Procedure for the spectrophotometric titrations}

The effect of anions upon the absorbance of receptors 4 and 8 was examined by adding $3 \mu \mathrm{L}$ of a $5 \times 10^{-3} \mathrm{M}$ TBAX solution to a known volume $(3 \mathrm{~mL})$ of a $1 \times 10^{-5} \mathrm{M}$ receptor solution. Some titrations were repeated adding $3 \mu \mathrm{L}$ of a $1 \times 10^{-3} \mathrm{M}$ TBAX (X = $\mathrm{H}_{2} \mathrm{PO}_{4}{ }^{-}$and $\mathrm{HP}_{2} \mathrm{O}_{3}{ }^{-}$) solution.

\section{Procedure for the NMR titrations}

The effect of anions upon the chemical shift in the signals of the receptors was examined by adding $5 \mu \mathrm{L}$ of $0.1 \mathrm{M}$ TBAX stock solution to a known volume $(0.5 \mathrm{~mL})$ of a $5 \times 10^{-3} \mathrm{M}$ receptor solution contained in an NMR tube. The addition was limited to $0.050 \mathrm{~mL}$, so that the dilution remained insignificant.

\section{Synthesis}

Synthesis of oligomethylene bis(2-aminobenzamides). In a round-bottom flask was added isatoic anhydride 1 (2.2 equiv., $18.30 \mathrm{mmol}$ ) in dry THF under an argon atmosphere. Then, alkyldiamine (1 equiv., $8.3 \mathrm{mmol}$ ) was added slowly to the anhydride mixture under stirring. The mixture was stirred for $24 \mathrm{~h}$ at reflux temperature. Once the reaction was completed, the solvent was removed by heating under vacuum. The solid was washed and filtered as many times as needed to give an analytically pure compound.

$N, N^{\prime}$-(Ethane-1,2-diyl)bis(2-aminobenzamide) (2a). Beige solid, 80.6\% yield. Mp 235-237 ${ }^{\circ} \mathrm{C}$. FTIR: 3473, 3367, 3277, 3057, 2944, 1626, 1580, $1540 \mathrm{~cm}^{-1} .{ }^{1} \mathrm{H}-\mathrm{NMR}\left(400 \mathrm{MHz}, \mathrm{DMSO}-d_{6}\right): \delta 8.29(\mathrm{~s}$, $2 \mathrm{H}), 7.48(\mathrm{~d}, J=8.0 \mathrm{~Hz}, 2 \mathrm{H}), 7.13(\mathrm{t}, J=8.0 \mathrm{~Hz}, 2 \mathrm{H}), 6.69(\mathrm{~d}, J=$ $8.0 \mathrm{~Hz}, 2 \mathrm{H}), 6.50(\mathrm{t}, J=8.0 \mathrm{~Hz}, 2 \mathrm{H}), 6.38(\mathrm{~s}, 4 \mathrm{H}), 3.47-3.22(\mathrm{~m}$, $4 \mathrm{H}) .{ }^{13} \mathrm{C}-\mathrm{NMR}\left(101 \mathrm{MHz}, \mathrm{DMSO}-d_{6}\right): \delta 169.1,149.6,131.6,128.1$, 116.3, 114.8, 114.5, 36.0. HRMS $\left(\mathrm{FAB}^{+}\right)$calculated for $\mathrm{C}_{16} \mathrm{H}_{19} \mathrm{~N}_{4} \mathrm{O}_{2}$ : 299.1508; found 299.1509. 
N,N'-(Propane-1,3-diyl)bis(3-aminobenzamide) (2b). White solid, 40.8\% yield. Mp 158-160 ${ }^{\circ}$ C. FTIR: $3470,3355,3294,3055$, 2967, 2931, 1625, 1576, $1531 \mathrm{~cm}^{-1}$. ${ }^{1} \mathrm{H}-\mathrm{NMR}$ (400 MHz, DMSO$\left.d_{6}\right): \delta 8.21(\mathrm{~s}, 2 \mathrm{H}), 7.47(\mathrm{~d}, J=7.7 \mathrm{~Hz}, 2 \mathrm{H}), 7.13(\mathrm{t}, J=7.2 \mathrm{~Hz}, 2 \mathrm{H})$, $6.69(\mathrm{~d}, J=7.7 \mathrm{~Hz}, 2 \mathrm{H}), 6.51(\mathrm{t}, J=7.2 \mathrm{~Hz}, 2 \mathrm{H}), 6.37(\mathrm{~s}, 4 \mathrm{H}), 3.27$ (brs, 4H), 1.81-1.61 (m, 2H). ${ }^{13} \mathrm{C}-\mathrm{NMR}$ (101 MHz, DMSO- $\left.d_{6}\right)$ : $\delta$ 168.8, 149.4, 131.4, 127.8, 116.2, 114.8, 114.5, 36.5, 29.2. HRMS $\left(\mathrm{FAB}^{+}\right)$calculated for $\mathrm{C}_{17} \mathrm{H}_{21} \mathrm{~N}_{4} \mathrm{O}_{2}$ : 313.1665; found 313.1699.

N,N'-(Butane-1,4-diyl)bis(3-aminobenzamide) (2c). White solid, $72.2 \%$ yield. Mp 190-192 ${ }^{\circ} \mathrm{C}$. FTIR: 3469, 3557, 3284, 3056, 2930 , 2860, 1621, 1578, $1534 \mathrm{~cm}^{-1}$. ${ }^{1} \mathrm{H}-\mathrm{NMR}$ (400 MHz, DMSO- $d_{6}$ ): $\delta 8.19(\mathrm{t}, J=4.9 \mathrm{~Hz}, 2 \mathrm{H}), 7.46(\mathrm{~d}, J=7.85 \mathrm{~Hz}, 2 \mathrm{H}), 7.12(\mathrm{t}, J=$ $7.4 \mathrm{~Hz}, 2 \mathrm{H}), 6.68(\mathrm{~d}, J=7.85 \mathrm{~Hz}, 2 \mathrm{H}), 6.49(\mathrm{t}, J=7.4 \mathrm{~Hz}, 2 \mathrm{H}), 6.36$ (brs, 4H), 3.40-3.18 (m, 4H), 1.59-1.49 (m, 4H). ${ }^{13} \mathrm{C}-\mathrm{NMR}$ (101 MHz, DMSO- $\left.d_{6}\right): \delta 168.7,149.4,131.3,127.9,116.2,114.9,114.4$, 38.4, 26.7. HRMS $\left(\mathrm{FAB}^{+}\right)$calculated for $\mathrm{C}_{18} \mathrm{H}_{23} \mathrm{~N}_{4} \mathrm{O}_{2}$ : 327.1821; found 327.1820 .

N,N'-(Hexane-1,6-diyl)bis(3-aminobenzamide) (2d). White solid, $54.8 \%$ yield. Mp 158-160 ${ }^{\circ}$ C. FTIR: 3473 , 3668, 3292, 3057, 2926, 2858, 1622, 1581, $1538 \mathrm{~cm}^{-1}$. ${ }^{1} \mathrm{H}-\mathrm{NMR}$ (400 MHz, DMSO$\left.d_{6}\right): \delta 8.16(\mathrm{t}, J=4.4 \mathrm{~Hz}, 2 \mathrm{H}), 7.45(\mathrm{~d}, J=7.9 \mathrm{~Hz}, 2 \mathrm{H}), 7.11(\mathrm{t}, J=$ $7.4 \mathrm{~Hz}, 2 \mathrm{H}), 6.67(\mathrm{~d}, J=7.9 \mathrm{~Hz}, 2 \mathrm{H}), 6.50(\mathrm{t}, J=7.4 \mathrm{~Hz}, 2 \mathrm{H}), 6.34$ (brs, $4 \mathrm{H}), 3.28-3.10(\mathrm{~m}, 4 \mathrm{H}), 1.59-1.41(\mathrm{~m}, 4 \mathrm{H}), 1.41-1.22(\mathrm{~m}$, $4 \mathrm{H}) .{ }^{13} \mathrm{C}-\mathrm{NMR}\left(101 \mathrm{MHz}, \mathrm{DMSO}-d_{6}\right): \delta 169.2,150.0,131.9,128.5$, 116.7, 115.6, 115.0, 39.2, 29.7, 26.8.

N,N'-(Octane-1,8-diyl)bis(3-aminobenzamide) (2e). Pearl solid, $36.1 \%$ yield. Mp 164-166 ${ }^{\circ} \mathrm{C}$. FTIR: 3475 , 3670, 3292, 3057, 2926, 2858, 1622, 1581, $1538 \mathrm{~cm}^{-1}$. ${ }^{1} \mathrm{H}-\mathrm{NMR}$ (400 MHz, DMSO- $d_{6}$ ): $\delta 8.15(\mathrm{t}, J=4.9 \mathrm{~Hz}, 2 \mathrm{H}), 7.45(\mathrm{~d}, J=7.9 \mathrm{~Hz}, 2 \mathrm{H}), 7.11(\mathrm{t}, J=$ $7.4 \mathrm{~Hz}, 2 \mathrm{H}), 6.67(\mathrm{~d}, J=7.9 \mathrm{~Hz}, 2 \mathrm{H}), 6.49(\mathrm{t}, J=7.4 \mathrm{~Hz}, 2 \mathrm{H}), 6.35$ (brs, $4 \mathrm{H}), 3.19$ (q, $J=6.6 \mathrm{~Hz}, 4 \mathrm{H}), 1.56-1.41$ (m, 4H), 1.36-1.19 $(\mathrm{m}, 8 \mathrm{H}) .{ }^{13} \mathrm{C}-\mathrm{NMR}\left(101 \mathrm{MHz}, \mathrm{DMSO}-d_{6}\right): \delta 169.2,150.0,131.9$, 128.5 116.7 , 115.6, 115.0, 39.3, 29.6, 29.3, 27.0. $\mathrm{EM}\left(\mathrm{FAB}^{+}\right): 383$ $[\mathrm{M}+\mathrm{H}]^{+}$. HRMS $\left(\mathrm{FAB}^{+}\right)$calculated for $\mathrm{C}_{22} \mathrm{H}_{31} \mathrm{~N}_{4} \mathrm{O}_{2}$ : 383.2447; found: 383.2469 .

Synthesis of oligomethylene bis(2-nitrophenylureylbenzamide). In a round-bottom flask was dissolved the intermediate 2 ( 1 equiv., $1.70 \mathrm{mmol}$ ) in dry THF under an $\mathrm{Ar}$ atmosphere. Then, 1-isocyanate-4-nitrobenzene 3 (2.2 equiv., $2.81 \mathrm{mmol}$ ) was slowly added to the mixture under stirring. The mixture was stirred for $24 \mathrm{~h}$ at reflux temperature. When the reaction was completed, the solvent was removed by heating under vacuum to obtain a solid, which was washed with different solvents and filtered as many times as needed to give an analytically pure compound.

$N, N^{\prime}$-(Ethane-1,2-diyl)bis(2-(3-(4-nitrophenyl)ureyl)benzamide) (4a). Yellow solid, 92.9\% yield. Mp 214-216 ${ }^{\circ}$ C. FTIR: 3290, 3218, 3157, 3096, 2934, 2872, 1699, 1629, 1562, $1497 \mathrm{~cm}^{-1} \cdot{ }^{1} \mathrm{H}-$ NMR (400 MHz, DMSO- $\left.d_{6}\right): \delta 10.55(\mathrm{~s}, 2 \mathrm{H}), 10.43(\mathrm{~s}, 2 \mathrm{H}), 8.85$ (brs, $2 \mathrm{H}), 8.20(\mathrm{~d}, J=9.3 \mathrm{~Hz}, 4 \mathrm{H}), 8.20(\mathrm{dd}, J=8.1,1.0 \mathrm{~Hz}, 2 \mathrm{H})$, $7.75(\mathrm{~d}, J=9.3 \mathrm{~Hz}, 4 \mathrm{H}), 7.71(\mathrm{dd}, J=8.1,1.5 \mathrm{~Hz}, 2 \mathrm{H}), 7.43(\mathrm{t}, J=$ $8.3 \mathrm{~Hz}, 2 \mathrm{H}), 7.06(\mathrm{t}, J=8.3 \mathrm{~Hz}, 2 \mathrm{H}), 3.57-3.49(\mathrm{~m}, 4 \mathrm{H}) .{ }^{13} \mathrm{C}-\mathrm{NMR}$ (101 MHz, DMSO- $d_{6}$ ): $\delta 168.4,151.8,146.6,140.9,139.0,131.4$, 128.0, 124.9, 121.6, 121.2, 120.7, 117.6, 25.0. HRMS $\left(\mathrm{FAB}^{+}\right)$ calculated for $\mathrm{C}_{30} \mathrm{H}_{27} \mathrm{~N}_{8} \mathrm{O}_{8}$ : 627.5940; found 627.5919.
$N, N^{\prime}$-(Propane-1,3-diyl)bis(3-(3-(4-nitrophenyl)ureyl)benzamide) (4c). Yellow solid, $84.8 \%$ yield. Mp 218-220 ${ }^{\circ}$ C. FTIR: 3278, 3218, 3155, 3092, 2924, 2855, 1688, 1616, 1560, $1493 \mathrm{~cm}^{-1} .{ }^{1} \mathrm{H}-\mathrm{NMR}$ $\left(400 \mathrm{MHz}, \mathrm{DMSO}-d_{6}\right): \delta 10.58(\mathrm{~s}, 2 \mathrm{H}), 10.41(\mathrm{~s}, 2 \mathrm{H}), 8.77(\mathrm{t}, J=$ $5.3 \mathrm{~Hz}, 2 \mathrm{H}), 8.24(\mathrm{dd}, J=7.8,1.2 \mathrm{~Hz}, 2 \mathrm{H}), 8.18(\mathrm{~d}, J=9.3 \mathrm{~Hz}$, $4 \mathrm{H}), 7.75(\mathrm{~d}, J=9.3 \mathrm{~Hz}, 4 \mathrm{H}), 7.70(\mathrm{dd}, J=7.8,1.2 \mathrm{~Hz}, 2 \mathrm{H}), 7.48(\mathrm{t}$, $J=7.3 \mathrm{~Hz}, 2 \mathrm{H}), 7.10(\mathrm{t}, J=7.5 \mathrm{~Hz}, 2 \mathrm{H}), 3.40(\mathrm{q}, J=6.4 \mathrm{~Hz}, 4 \mathrm{H})$, 1.88 (qnt, $J=6.9 \mathrm{~Hz}, 2 \mathrm{H}$ ). ${ }^{13} \mathrm{C}-\mathrm{NMR}$ (101 MHz, DMSO- $d_{6}$ ): $\delta 168.2,151.8,146.5,140.9,139.0,131.4,127.8,124.9,121.6$, 121.1, 120.7, 117.6, 28.7, 26.0. HRMS $\left(\mathrm{FAB}^{+}\right)$calculated for $\mathrm{C}_{31} \mathrm{H}_{29} \mathrm{~N}_{8} \mathrm{O}_{8}$ : 641.2108; found: 641.2122.

$N, N^{\prime}$-(Butane-1,4-diyl)bis(3-(3-(4-nitrophenyl)ureyl)benzamide) (4c). Yellow solid, 81.6\% yield. Mp 230-232 ${ }^{\circ} \mathrm{C}$. FTIR: 3268, 3213, 3154, 3087, 2949, 2871, 1693, 1617, 1561, $1496 \mathrm{~cm}^{-1} \cdot{ }^{1} \mathrm{H}-\mathrm{NMR}$ $\left(400 \mathrm{MHz}, \mathrm{DMSO}-d_{6}\right): \delta 10.62(\mathrm{~s}, 2 \mathrm{H}), 10.42(\mathrm{~s}, 2 \mathrm{H}), 8.76(\mathrm{t}, J=$ $5.5 \mathrm{~Hz}, 2 \mathrm{H}), 8.24(\mathrm{dd}, J=8.5,0.9 \mathrm{~Hz}, 2 \mathrm{H}), 8.19(\mathrm{~d}, J=9.3 \mathrm{~Hz}$, $4 \mathrm{H}), 7.76(\mathrm{~d}, J=9.3 \mathrm{~Hz}, 4 \mathrm{H}), 7.68(\mathrm{dd}, J=7.8,1.5 \mathrm{~Hz}, 2 \mathrm{H}), 7.46(\mathrm{t}$, $J=8.5 \mathrm{~Hz}, 1 \mathrm{H}), 7.08(\mathrm{t}, J=7.8 \mathrm{~Hz}, 1 \mathrm{H}), 3.43-3.23(\mathrm{~m}, 4 \mathrm{H}), 1.69-$ $1.55(\mathrm{~m}, 4 \mathrm{H}) .{ }^{13} \mathrm{C}-\mathrm{NMR}$ (101 MHz, DMSO- $\left.d_{6}\right): \delta$ 168.2, 151.9, $146.7,141.0$, 139.1, 131.5, 127.8, 125.0, 121.6, 121.2, 120.7, 117.7, 26.5, 25.1. HRMS $\left(\mathrm{FAB}^{+}\right)$calculated for $\mathrm{C}_{32} \mathrm{H}_{31} \mathrm{~N}_{8} \mathrm{O}_{8}$ : 655.2265; found: 655.2276 .

N,N'-(Hexane-1,6-diyl)bis(3-(3-(4-nitrophenyl)ureyl)benzamide) (4d). Yellow solid, 91.9\% yield. Mp 236-238 ${ }^{\circ}$ C. FT-IR: 3276, 3216, 3155, 3090, 2964, 2868, 1697, 1621, 1564, $1497 \mathrm{~cm}^{-1} \cdot{ }^{1} \mathrm{H}-$ NMR (400 MHz, DMSO- $\left.d_{6}\right): \delta 10.60(\mathrm{~s}, 2 \mathrm{H}), 10.41(\mathrm{~s}, 2 \mathrm{H}), 8.72(\mathrm{t}$, $J=5.5 \mathrm{~Hz}, 2 \mathrm{H}), 8.23(\mathrm{~d}, J=7.8 \mathrm{~Hz}, 2 \mathrm{H}), 8.18(\mathrm{~d}, J=9.3 \mathrm{~Hz}, 4 \mathrm{H})$, $7.76(\mathrm{~d}, J=9.3 \mathrm{~Hz}, 4 \mathrm{H}), 7.67(\mathrm{dd}, J=7.8,1.2 \mathrm{~Hz}, 2 \mathrm{H}), 7.46(\mathrm{t}, J=$ $8.1 \mathrm{~Hz}, 2 \mathrm{H}), 7.09$ (t, $J=8.1 \mathrm{~Hz}, 2 \mathrm{H}), 3.29$ (q, $J=6.6 \mathrm{~Hz}, 4 \mathrm{H}), 1.65-$ $1.49(\mathrm{~m}, 4 \mathrm{H}), 1.44-1.30(\mathrm{~m}, 4 \mathrm{H}) .{ }^{13} \mathrm{C}-\mathrm{NMR}\left(101 \mathrm{MHz}, \mathrm{DMSO}-d_{6}\right)$ : $\delta 168.2,151.9,146.7,141.1,139.1,131.4,127.8,125.0,121.7$, 121.4, 120.8, 117.7, 28.9, 26.3, 25.1.

$N, N^{\prime}$-(Octane-1,8-diyl)bis(3-(3-(4-nitrophenyl)ureyl)benzamide) (4e). Brown solid, $96.0 \%$ yield. Mp 236-238 ${ }^{\circ} \mathrm{C}$. FTIR: 32783218 , 3155, 3092, 2924, 2855, 1688, 1616, $1560 \mathrm{~cm}^{-1} .{ }^{1} \mathrm{H}-\mathrm{NMR}$ (400 MHz, DMSO- $\left.d_{6}\right): \delta 10.59(\mathrm{~s}, 2 \mathrm{H}), 10.41(\mathrm{~s}, 2 \mathrm{H}), 8.70(\mathrm{t}, J=5.6 \mathrm{~Hz}$, $2 \mathrm{H}), 8.23(\mathrm{dd}, J=8.4,1.0 \mathrm{~Hz}, 2 \mathrm{H}), 8.19$ (d, $J=9.3 \mathrm{~Hz}, 4 \mathrm{H}), 7.76$ $(\mathrm{d}, J=9.3 \mathrm{~Hz}, 4 \mathrm{H}), 7.66(\mathrm{dd}, J=8.0,1.3 \mathrm{~Hz}, 2 \mathrm{H}), 7.47(\mathrm{t}, J=$ $8.4 \mathrm{~Hz}, 2 \mathrm{H}), 7.09$ (t, $J=8.0 \mathrm{~Hz}, 2 \mathrm{H}), 3.27$ (q, $J=6.8 \mathrm{~Hz}, 4 \mathrm{H}), 1.62-$ $1.47(\mathrm{~m}, 4 \mathrm{H}), 1.40-1.22(\mathrm{~m}, 8 \mathrm{H}) .{ }^{13} \mathrm{C}-\mathrm{NMR}$ (101 MHz, DMSO- $\left.d_{6}\right)$ : $\delta 168.1,151.9,146.7,141.0,139.0,131.4,127.8,125.0,121.7$, 121.4, 120.7, 117.7, 39.2, 28.8, 26.5. HRMS $\left(\mathrm{FAB}^{+}\right)$calculated for $\mathrm{C}_{36} \mathrm{H}_{39} \mathrm{~N}_{8} \mathrm{O}_{8}$ : 711.2891; found: 711.2901.

Synthesis of oligomethylene bis(3-nitrophenylbenzamides). In a $100 \mathrm{~mL}$ round-bottom flask with magnetic stirring, 3nitrobenzoyl chloride (2.2 equiv.) was dissolved in $22 \mathrm{~mL}$ of dry THF and stirred for 15 min under an $\mathrm{Ar}$ atmosphere. Then, $\mathrm{Et}_{3} \mathrm{~N}$ $(0.3 \mathrm{~mL})$ and dialkylamine (1.0 equiv.) were added dropwise, and the reaction mixture was stirred for $12 \mathrm{~h}$. The solvent was removed by heating under vacuum. The solid obtained was washed with different solvents and filtered as many times as needed to give an analytically pure solid.

N,N'-(Ethane-1,2-diyl)bis(3-(3-(4-nitrophenyl)benzamide)) (6a). White solid, $88.7 \%$ yield. Mp 216-218 ${ }^{\circ} \mathrm{C}$. FTIR: 3300, 3079, 2947, 2869, 1637, 1551, $1453 \mathrm{~cm}^{-1}$. ${ }^{1} \mathrm{H}-\mathrm{NMR}$ (400 MHz, DMSO$\left.d_{6}\right): \delta 9.08(\mathrm{~s}, 2 \mathrm{H}), 8.69(\mathrm{t}, J=2.2 \mathrm{~Hz}, 2 \mathrm{H}), 8.38(\mathrm{ddd}, J=8.2,2.2$, 
$1.2 \mathrm{~Hz}, 2 \mathrm{H}), 8.32(\mathrm{td}, J=7.8,1.2 \mathrm{~Hz}, 2 \mathrm{H}), 7.79(\mathrm{t}, J=8.2 \mathrm{~Hz}, 2 \mathrm{H})$, 3.57-3.48 (m, 4H). ${ }^{13} \mathrm{C}-\mathrm{NMR}$ (101 MHz, DMSO- $\left.d_{6}\right): \delta$ 164.3, 147.6, 135.9, 133.6, 130.0, 125.7, 121.9, 36.5. $\mathrm{MS}\left(\mathrm{FAB}^{+}\right) \mathrm{m} / z: 359$ $[\mathrm{M}+\mathrm{H}]^{+}$. HRMS $\left(\mathrm{FAB}^{+}\right)$calculated for $\mathrm{C}_{16} \mathrm{H}_{15} \mathrm{~N}_{4} \mathrm{O}_{6}$ : 359.0992; found: 359.0981 .

N,N'-(Propane-1,3-diyl)bis(3-(3-(4-nitrophenyl)benzamide)) (6b). White solid, 90.5\% yield. Mp 210-212 ${ }^{\circ} \mathrm{C}$. FTIR: 3363, 3098, 2945, 2874, 1637, 1548, 1524, $1471 \mathrm{~cm}^{-1} .{ }^{1} \mathrm{H}-\mathrm{NMR}(400 \mathrm{MHz}$, DMSO- $\left.d_{6}\right): \delta 8.93(\mathrm{t}, J=5.5 \mathrm{~Hz}, 2 \mathrm{H}), 8.68(\mathrm{t}, J=2.0 \mathrm{~Hz}, 2 \mathrm{H}), 8.38$ (ddd, $J=8.0,2.0,1.0 \mathrm{~Hz}, 2 \mathrm{H}), 8.31(\mathrm{td}, J=8.0,2.0 \mathrm{~Hz}, 2 \mathrm{H}), 7.78$ (t, $J=8.0 \mathrm{~Hz}, 2 \mathrm{H}), 3.46-3.36(\mathrm{~m}, 4 \mathrm{H}), 1.87$ (qnt, $J=6.9 \mathrm{~Hz}, 2 \mathrm{H}$ ). ${ }^{13} \mathrm{C}-\mathrm{NMR}\left(101 \mathrm{MHz}, \mathrm{DMSO}-d_{6}\right): \delta$ 164.0, 147.7, 135.9, 133.5, 130.0, 125.7, 121.8, 37.4, 28.7. $\mathrm{MS}\left(\mathrm{FAB}^{+}\right) \mathrm{m} / \mathrm{z}: 373[\mathrm{M}+\mathrm{H}]^{+}$. HRMS $\left(\mathrm{FAB}^{+}\right)$calculated for $\mathrm{C}_{17} \mathrm{H}_{17} \mathrm{~N}_{4} \mathrm{O}_{6}$ : 373.1148; found: 373.1143.

N,N'-(Butane-1,4-diyl)bis(3-(3-(4-nitrophenyl)benzamide)) (6c). White solid, $80.3 \%$ yield. Mp 242-244 ${ }^{\circ} \mathrm{C}$. FTIR: 3361, 3091, 2937, 2861, 1637, 1551, 1523, $1478 \mathrm{~cm}^{-1} .{ }^{1} \mathrm{H}-\mathrm{NMR}(400 \mathrm{MHz}$, DMSO- $\left.d_{6}\right): \delta 8.87(\mathrm{t}, J=5.4 \mathrm{~Hz}, 2 \mathrm{H}), 8.67(\mathrm{t}, J=2.0 \mathrm{~Hz}, 2 \mathrm{H}), 8.37$ $(\mathrm{ddd}, J=0.7,2.0,8.1 \mathrm{~Hz}, 1 \mathrm{H}), 8.29(\mathrm{td}, J=1.2,8.1 \mathrm{~Hz}, 2 \mathrm{H}), 7.77$ $(\mathrm{t}, J=8.1 \mathrm{~Hz}, 2 \mathrm{H}), 3.39-3.26(\mathrm{~m}, 4 \mathrm{H}), 1.66-1.56(\mathrm{~m}, 4 \mathrm{H}) .{ }^{13} \mathrm{C}-$ NMR (101 MHz, DMSO- $\left.d_{6}\right): \delta$ 163.8, 147.6, 135.9, 133.5, 130.0, 125.6, 121.7, 33.6, 26.4. MS $\left(\mathrm{FAB}^{+}\right) \mathrm{m} / z: 387[\mathrm{M}+\mathrm{H}]^{+}$. HRMS $\left(\mathrm{FAB}^{+}\right)$calculated for $\mathrm{C}_{18} \mathrm{H}_{19} \mathrm{~N}_{4} \mathrm{O}_{6}: 387.1305$; found: 387.1310 .

N,N'-(Hexane-1,6-diyl)bis(3-(3-(4-nitrophenyl)benzamide)) (6d). White solid, 59\% yield. Mp 192-194 ${ }^{\circ} \mathrm{C}$. FTIR: 3309, 3100, 2935, 2873, 1629, 1577, 1530, $1478 \mathrm{~cm}^{-1}$. ${ }^{1} \mathrm{H}-\mathrm{NMR}$ (400 MHz, DMSO$\left.d_{6}\right): \delta 8.83(\mathrm{t}, J=5.4 \mathrm{~Hz}, 2 \mathrm{H}), 8.67(\mathrm{t}, J=2.0 \mathrm{~Hz}, 2 \mathrm{H}), 8.37$ (ddd, $J$ $=8.1,2.3,1.0 \mathrm{~Hz}, 2 \mathrm{H}), 8.28(\mathrm{td}, J=8.1,1.3 \mathrm{~Hz}, 2 \mathrm{H}), 7.77(\mathrm{t}, J=$ $8.1 \mathrm{~Hz}, 2 \mathrm{H}), 3.38-3.24(\mathrm{~m}, 4 \mathrm{H}), 1.63-1.47$ (m, 4H), 1.46-1.30 (m, $4 \mathrm{H}) .{ }^{13} \mathrm{C}-\mathrm{NMR}\left(101 \mathrm{MHz}, \mathrm{DMSO}-d_{6}\right): \delta 163.8,147.7,135.9,133.5$, 130.0, 125.6, 121.8, 39.3, 28.8, 26.1. $\mathrm{MS}\left(\mathrm{FAB}^{+}\right) \mathrm{m} / z: 415[\mathrm{M}+\mathrm{H}]^{+}$. HRMS $\left(\mathrm{FAB}^{+}\right)$calculated for $\mathrm{C}_{20} \mathrm{H}_{23} \mathrm{~N}_{4} \mathrm{O}_{6}$ : 415.4260; found: 415.4268.

N,N'-(Octane-1,8-diyl)bis(3-(3-(4-nitrophenyl)benzamide)) (6e). White solid, $59.0 \%$ yield. Mp $178-180{ }^{\circ} \mathrm{C}$. FTIR: 3312, 3100, 2932, 2872, 1629, 1577, 1530, $1477 \mathrm{~cm}^{-1} \cdot{ }^{1} \mathrm{H}-\mathrm{NMR}(400 \mathrm{MHz}$, DMSO- $\left.d_{6}\right): \delta 8.81(\mathrm{~s}, 2 \mathrm{H}), 8.66(\mathrm{~s}, 2 \mathrm{H}), 8.28(\mathrm{~d}, J=7.3 \mathrm{~Hz}, 2 \mathrm{H})$, $8.37(\mathrm{~d}, J=8.1 \mathrm{~Hz}, 2 \mathrm{H}), 7.77(\mathrm{t}, J=7.3 \mathrm{~Hz}, 2 \mathrm{H}), 1.55(\mathrm{~m}, 4 \mathrm{H})$, 1.43-1.17 (m, 8H).

Synthesis of oligomethylene bis(3-aminobenzamides). In a $100 \mathrm{~mL}$ round-bottom flask with magnetic stirring, $\mathrm{Pd}(10 \%) / \mathrm{C}$ ( 0.1 equiv.) and the oligomethylene bis(3-nitrobenzamide) were added. The air in the flask was purged with $\mathrm{Ar}$ and $\mathrm{MeOH}$ was added $(10 \mathrm{~mL} \times 1 \mathrm{mmol})$. The mixture was stirred for $20 \mathrm{~min}$ and then the Ar was purged with $\mathrm{H}_{2}$ to ensure a pure atmosphere in the flask. Then, a balloon filled with $\mathrm{H}_{2}$ was connected to the flask and the reaction mixture was stirred for $24 \mathrm{~h}$. Finally, the mixture was filtered to separate the catalyst and the solvent was removed by heating under vacuum to obtain the product.

$N, N^{\prime}$-(Ethane-1,2-diyl)bis(3-aminobenzamide) (7a). Yellow solid, 42.0\% yield. Mp 280-282 ${ }^{\circ}$ C. FTIR: 3456, 3330, 3216, 3056, 2949, 2912, 1632, 1579, 1537, $1486 \mathrm{~cm}^{-1} .{ }^{1} \mathrm{H}-\mathrm{NMR}(200 \mathrm{MHz}$, DMSO- $d_{6}$ ): $\delta 8.33$ (brs, 2H), 7.07 (t, $\left.J=7.7 \mathrm{~Hz}, 2 \mathrm{H}\right), 7.04$ (brs, $2 \mathrm{H}), 6.95(\mathrm{dd}, J=7.8,1.3 \mathrm{~Hz}, 2 \mathrm{H}), 6.69(\mathrm{ddd}, J=7.8,2.1,1.0 \mathrm{~Hz}$, 2H), 5.20 (brs, 4H), 3.64-3.21 (m, 4H). ${ }^{13} \mathrm{C}-\mathrm{NMR}(50 \mathrm{MHz}$,
DMSO- $\left.d_{6}\right): \delta 167.0,148.4,135.1,128.3,116.1,114.0,112.6,37.5$. MS $\left(\mathrm{FAB}^{+}\right) m / z: 299[\mathrm{M}+\mathrm{H}]^{+}$. HRMS $\left(\mathrm{FAB}^{+}\right)$calculated for $\mathrm{C}_{16} \mathrm{H}_{19} \mathrm{~N}_{4} \mathrm{O}_{2}$ : 299.1508; found: 299.1509.

$N, N^{\prime}$-(Propane-1,3-diyl)bis(3-aminobenzamide) (7b). Brown solid, $97.8 \%$ yield. Mp 98-100 ${ }^{\circ} \mathrm{C}$. FTIR: 3324, 3226, 3063, 2931, 1622, 1579, 1528, $1487 \mathrm{~cm}^{-1}$. ${ }^{1} \mathrm{H}-\mathrm{NMR}$ (200 MHz, DMSO- $\left.d_{6}\right)$ : $\delta 8.29(\mathrm{t}, J=5.7 \mathrm{~Hz}, 2 \mathrm{H}), 7.08(\mathrm{t}, J=7.7 \mathrm{~Hz}, 2 \mathrm{H}), 7.05(\mathrm{~s}, 2 \mathrm{H}), 6.96$ $(\mathrm{d}, J=7.7 \mathrm{~Hz}, 2 \mathrm{H}), 6.69$ (ddd, $J=7.7,2.4,1.0 \mathrm{~Hz}, 2 \mathrm{H}), 5.22$ (brs, $4 \mathrm{H}), 3.35-3.20(\mathrm{~m}, 4 \mathrm{H}) .{ }^{13} \mathrm{C}-\mathrm{NMR}\left(50 \mathrm{MHz}\right.$, DMSO- $\left.d_{6}\right): \delta 167.5$, 149.1, 136.0, 129.1 116.8, 114.6, 113.2, 37.2, 29.8. $\mathrm{MS}_{\left(\mathrm{FAB}^{+}\right) \mathrm{m} /}$ $z: 313[\mathrm{M}+\mathrm{H}]^{+}$. HRMS $\left(\mathrm{FAB}^{+}\right)$calculated for $\mathrm{C}_{17} \mathrm{H}_{21} \mathrm{~N}_{4} \mathrm{O}_{2}$ : 313.1665; found: 312.1699.

$N, N^{\prime}$-(Butane-1,4-diyl)bis(3-aminobenzamide) (7c). Orange solid, $69.2 \%$ yield. Mp 120-122 ${ }^{\circ}$ C. FTIR: 3433, 3332, 3222, 3057 , 2943, 2866, 1647, 1583, 1547, $1520 \mathrm{~cm}^{-1} .{ }^{1} \mathrm{H}-\mathrm{NMR}(200 \mathrm{MHz}$, DMSO- $\left.d_{6}\right): \delta 8.23(\mathrm{t}, J=5.5 \mathrm{~Hz}, 2 \mathrm{H}), 7.04(\mathrm{t}, J=7.5 \mathrm{~Hz}, 2 \mathrm{H}), 7.01$ (s, 2H), 6.94 (ddd, $J=7.5,2.7,1.1 \mathrm{~Hz}, 2 \mathrm{H}), 6.67$ (ddd, $J=7.5,2.4$, $1.3 \mathrm{~Hz}, 2 \mathrm{H}), 5.21$ (brs, 4H), 3.32-3.12 (m, 4H), 1.52 (brs, 4H). MS $\left(\mathrm{CI}^{+}\right) \mathrm{m} / z: 326[\mathrm{M}+\mathrm{H}]^{+}$. HRMS $\left(\mathrm{FAB}^{+}\right)$calculated for $\mathrm{C}_{18} \mathrm{H}_{23} \mathrm{~N}_{4} \mathrm{O}_{2}$ : 327.1821; found: 327.1820 .

$N, N^{\prime}$-(Hexane-1,6-diyl)bis(3-aminobenzamide) (7d). Melon color solid, $80.3 \%$ yield. Mp 100-102 ${ }^{\circ} \mathrm{C}$. FTIR: 3390, 3324, 3218, 3054, 2928, 2854, 1625, 1582, 1533, $1489 \mathrm{~cm}^{-1} .{ }^{1} \mathrm{H}-\mathrm{NMR}(200$ MHz, DMSO- $\left.d_{6}\right): \delta 8.18(\mathrm{t}, J=5.6 \mathrm{~Hz}, 2 \mathrm{H}), 7.00(\mathrm{t}, J=7.6 \mathrm{~Hz}, 2 \mathrm{H})$, $7.01(\mathrm{~s}, 2 \mathrm{H}), 6.92(\mathrm{td}, J=7.6,0.5 \mathrm{~Hz}, 2 \mathrm{H}), 6.66(\mathrm{ddd}, J=7.6,2.0$, $1.0 \mathrm{~Hz}, 2 \mathrm{H}), 5.19$ (s, 4H), 3.31-3.08 (m, 4H), 1.63-1.40 (m, 4H), 1.31 (brs, $4 \mathrm{H}$ ). ${ }^{13} \mathrm{C}-\mathrm{NMR}$ (50 MHz, DMSO- $d_{6}$ ): $\delta$ 166.9, 148.6, 135.8, 128.5, 116.2, 114.2, 112.8, 39.0, 29.2, 26.3.

$N, N^{\prime}$-(Octane-1,8-diyl)bis(3-aminobenzamide) (7e). Beige solid, $337 \mathrm{mg}, 0.88 \mathrm{mmol}, 90.4 \%$ yield. Mp 120-122 ${ }^{\circ} \mathrm{C}$. FTIR: 3393, 3349, 3208, 3054, 2926, 2853, 1625, 1583, 1538, $1490 \mathrm{~cm}^{-1} \cdot{ }^{1} \mathrm{H}-$ NMR (200 MHz, DMSO- $d_{6}$ ): $\delta 8.17(\mathrm{t}, J=5.5 \mathrm{~Hz}, 2 \mathrm{H}), 7.15-6.85$ (d, $J=7.8 \mathrm{~Hz}, 2 \mathrm{H}), 7.01(\mathrm{~s}, 2 \mathrm{H}), 6.92(\mathrm{dd}, J=7.8,1.2 \mathrm{~Hz}, 2 \mathrm{H})$, $6.66(\mathrm{td}, J=7.8,1.1 \mathrm{~Hz}, 1 \mathrm{H}), 5.19$ (brs $4 \mathrm{H}), 3.19$ (q, $J=6.5 \mathrm{~Hz}$, $4 \mathrm{H}), 1.58-1.39$ (m, 4H), 1.28 (s, 8H). ${ }^{13} \mathrm{C}-\mathrm{NMR}$ (50 MHz, DMSO$\left.d_{6}\right): \delta 166.9,148.6,135.8,128.5,116.2,114.2,112.9,29.2,28.8$, 26.5. $\mathrm{MS}\left(\mathrm{FAB}^{+}\right) \mathrm{m} / z: 383[\mathrm{M}+\mathrm{H}]^{+}$. HRMS $\left(\mathrm{FAB}^{+}\right)$calculated for $\mathrm{C}_{22} \mathrm{H}_{31} \mathrm{~N}_{4} \mathrm{O}_{2}$ : 383.2447; found: 383.2469.

Synthesis de oligomethylene bis( $p$-nitrophenylureabenzamides). In a $100 \mathrm{~mL}$ round-bottom flask with magnetic stirring, the oligomethylene bis(3-aminobenzamide) (1.0 equiv.) and the 4-nitrophenyl isocyanate (2.2 equiv.) were dissolved in dry THF $(10 \mathrm{~mL} \times 1 \mathrm{mmol})$. The mixture was stirred for $24 \mathrm{~h}$ at RT under an argon atmosphere. Then, the solvent was removed by heating under vacuum. The solid obtained was washed with different solvents and filtered as many as needed times to give an analytically pure compound.

N,N'-(Propane-1,3-diyl)bis(3-(3-(4-nitrophenyl)ureyl)benzamide) (8b). Brown solid, 51.7\% yield. Mp 184-186 ${ }^{\circ} \mathrm{C}$. FTIR: 3288, $3088,1638,1559 \mathrm{~cm}^{-1} .{ }^{1} \mathrm{H}-\mathrm{NMR}$ (400 MHz, DMSO- $d_{6}$ ): $\delta 9.47(\mathrm{~s}$, $2 \mathrm{H}), 9.08(\mathrm{~s}, 2 \mathrm{H}), 8.49(\mathrm{t}, J=5.6 \mathrm{~Hz}, 2 \mathrm{H}), 8.20(\mathrm{~d}, J=9.3 \mathrm{~Hz}, 4 \mathrm{H})$, $7.94(\mathrm{t}, J=1.7 \mathrm{~Hz}, 1 \mathrm{H}), 7.71(\mathrm{~d}, J=9.3 \mathrm{~Hz}, 4 \mathrm{H}), 7.64(\mathrm{ddd}, J=7.9$, 2.2, $1.2 \mathrm{~Hz}, 2 \mathrm{H}), 7.50(\mathrm{td}, J=7.9,1.2 \mathrm{~Hz}, 2 \mathrm{H}), 7.40(\mathrm{t}, J=7.9 \mathrm{~Hz}$, 2H), 3.59-3.03 (m, 4H), 1.79 (qnt, $J=6.8 \mathrm{~Hz}, 2 \mathrm{H}) .{ }^{13} \mathrm{C}-\mathrm{NMR}(101$ MHz, DMSO- $\left.d_{6}\right): \delta 166.1,151.9,146.2$, 141.0, 139.0, 135.4, 128.7, 125.0, 121.1, 120.1, 117.7, 117.5, 37.0, 29.2. $\mathrm{MS}\left(\mathrm{FAB}^{+}\right) \mathrm{m} / z: 641$ 

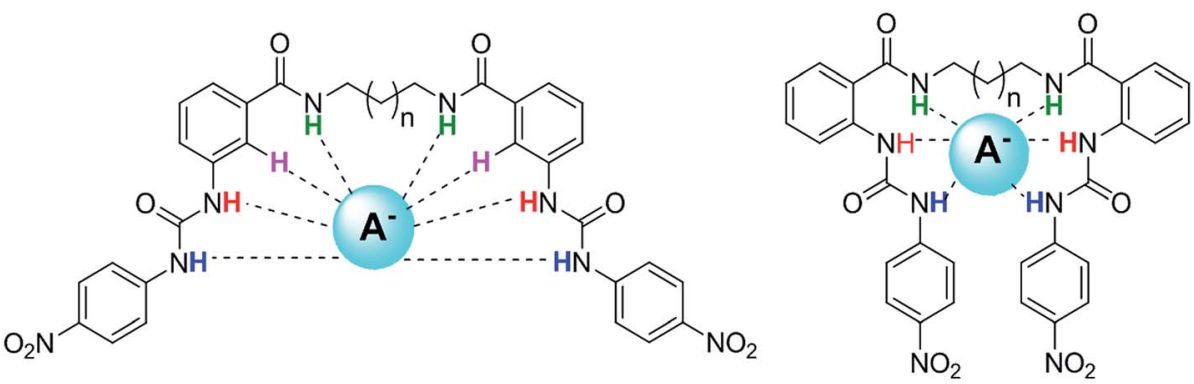

Fig. 1 Plausible interaction of oligomethylene bis(nitrophenylureylbenzamide) receptors with anions.

$[\mathrm{M}+\mathrm{H}]^{+}$. HRMS $\left(\mathrm{FAB}^{+}\right)$calculated for $\mathrm{C}_{31} \mathrm{H}_{29} \mathrm{~N}_{8} \mathrm{O}_{8}: 641.2108$; found: 641.2143 .

N,N'-(Buthane-1,4-diyl)bis(3-(3-(4-nitrophenyl)ureyl)benzamide) (8c). Pink solid, $77.0 \%$ yield. Mp 262-264 ${ }^{\circ} \mathrm{C}$. FTIR: 3256, 3058, 1633, $1535 \mathrm{~cm}^{-1} .{ }^{1} \mathrm{H}-\mathrm{NMR}$ (400 MHz, DMSO- $\left.d_{6}\right): \delta 9.45(\mathrm{~s}, 2 \mathrm{H})$, $9.06(\mathrm{~s}, 2 \mathrm{H}), 8.46(\mathrm{t}, J=5.5 \mathrm{~Hz}, 2 \mathrm{H}), 8.20(\mathrm{~d}, J=9.4 \mathrm{~Hz}, 4 \mathrm{H}), 7.91$ $(\mathrm{t}, J=1.8 \mathrm{~Hz}, 2 \mathrm{H}), 7.71(\mathrm{~d}, J=9.4 \mathrm{~Hz}, 4 \mathrm{H}), 7.63(\mathrm{ddd}, J=8.0,2.2$, $1.2 \mathrm{~Hz}, 2 \mathrm{H}), 7.49(\mathrm{td}, J=8.0,1.1 \mathrm{~Hz}, 2 \mathrm{H}), 7.39(\mathrm{t}, J=8.0 \mathrm{~Hz}, 2 \mathrm{H})$, 3.32-3.21 (m, 4H), 1.64-1.51 (m, 4H). ${ }^{13} \mathrm{C}-\mathrm{NMR}(101 \mathrm{MHz}$, DMSO- $\left.d_{6}\right): \delta 166.0,151.9,146.2,141.0,139.0,135.5,128.6$, 125.0, 121.0, 120.9, 117.8, 117.5, 38.9, 26.7. MS $\left(\mathrm{FAB}^{+}\right) \mathrm{m} / \mathrm{z}: 655$ $[\mathrm{M}+\mathrm{H}]^{+}$. HRMS $\left(\mathrm{FAB}^{+}\right)$calculated for $\mathrm{C}_{32} \mathrm{H}_{31} \mathrm{~N}_{8} \mathrm{O}_{8}$ : 655.2265; found: 656.2221 .

N,N'-(Hexane-1,6-diyl)bis(3-(3-(4-nitrophenyl)ureyl)benzamide) (8d). Yellow solid, 70.8\% yield. Mp 241-243 ${ }^{\circ}$ C. FTIR: 3328, 3093, 1624, $1550 \mathrm{~cm}^{-1}$. ${ }^{1} \mathrm{H}-\mathrm{NMR}\left(400 \mathrm{MHz}, \mathrm{DMSO}-d_{6}\right): \delta 9.45(\mathrm{~s}$, $2 \mathrm{H}), 9.05(\mathrm{~s}, 2 \mathrm{H}), 8.42(\mathrm{t}, J=5.1 \mathrm{~Hz}, 2 \mathrm{H}), 8.19(\mathrm{~d}, J=9.0 \mathrm{~Hz}, 4 \mathrm{H})$, $7.90(\mathrm{~s}, 2 \mathrm{H}), 7.70(\mathrm{~d}, J=9.0 \mathrm{~Hz}, 4 \mathrm{H}), 7.62(\mathrm{~d}, J=8.1 \mathrm{~Hz}, 2 \mathrm{H}), 7.47$ $(\mathrm{d}, J=7.6 \mathrm{~Hz}, 2 \mathrm{H}), 7.38(\mathrm{t}, J=7.8 \mathrm{~Hz}, 2 \mathrm{H}), 3.29-3.15(\mathrm{~m}, 4 \mathrm{H})$, 1.61-1.45 (m, 4H), 1.42-1.27 (m, 4H). MS $\left(\mathrm{FAB}^{+}\right) \mathrm{m} / \mathrm{z}: 683[\mathrm{M}+$ $\mathrm{H}]^{+}$. HRMS $\left(\mathrm{FAB}^{+}\right.$) calculated for $\mathrm{C}_{34} \mathrm{H}_{35} \mathrm{~N}_{8} \mathrm{O}_{8}$ : 683.2578; found: 683.2586 .

$N, N^{\prime}$-(Octane-1,8-diyl)bis(3-(3-(4-nitrophenyl)ureyl)benzamide)

(8e). Yellow solid, 97\% yield. Mp 266-268 ${ }^{\circ} \mathrm{C}$. FTIR: 3278, 3082, 2930, 2855, 1706, 1628, 1585, $1543 \mathrm{~cm}^{-1} .{ }^{1} \mathrm{H}-\mathrm{NMR}(400 \mathrm{MHz}$, DMSO- $\left.d_{6}\right): \delta 9.46(\mathrm{~s}, 2 \mathrm{H}), 9.06(\mathrm{~s}, 2 \mathrm{H}), 8.41(\mathrm{t}, J=5.5 \mathrm{~Hz}, 2 \mathrm{H})$, $8.20(\mathrm{~d}, J=9.3 \mathrm{~Hz}, 4 \mathrm{H}), 7.90(\mathrm{t}, J=1.8 \mathrm{~Hz}, 2 \mathrm{H}), 7.71$ (d, $J=$ $9.3 \mathrm{~Hz}, 4 \mathrm{H}), 7.63(\mathrm{ddd}, J=8.0,2.2,1.0 \mathrm{~Hz}, 2 \mathrm{H}), 7.47$ (td, $J=8.0$, $1.5 \mathrm{~Hz}, 2 \mathrm{H}), 7.38(\mathrm{t}, J=8.0 \mathrm{~Hz}, 2 \mathrm{H}), 3.29-3.19(\mathrm{~m}, 4 \mathrm{H}), 1.61-1.45$ $(\mathrm{m}, 4 \mathrm{H}), 1.32$ (s, 8H). ${ }^{13} \mathrm{C}-\mathrm{NMR}$ (101 MHz, DMSO- $\left.d_{6}\right): \delta 165.8$, $151.8,146.1,140.8,138.8,135.4,128.5,124.9,120.7,117.6$, $117.3,39.0,28.9,28.6,26.3$.

\section{Molecular modeling}

The molecular optimization of the geometries for the proposed complexes was carried out by DFT method, using B3LYP and the 6-31G(d) base in the Gaussian 09 computational program. ${ }^{24}$

\section{Results and discussion}

\section{Synthesis and characterization}

The synthesis of bis(ureas) $\mathbf{4}$ (ortho receptors) started with the reaction of isatoic anhydride (1) and an alkyldiamine (a: ethane1,2-diamine, b: propane-1,3-diamine, c: buthane-1,4-diamine, d: hexane-1,6-diamine, e: octane-1,8-diamine) in THF at reflux temperature to obtain the corresponding oligomethylene bis(2aminobenzamides) 2 with 81\%, 41\%, 72\%, 55\%, and 36\% yields, respectively. The intermediates 2 were directly reacted with 4-nitrophenyl isocyanate in THF to obtain receptors 4 in 93\%, 85\%, 82\%, 92\%, and 96\% yields, respectively (Scheme 1). An ortho mono-ureylbenzamide was synthetized under the same experimental conditions using propylamine in a $36 \%$ global yield.

In the FTIR spectra, stretching $\mathrm{N}-\mathrm{H}$ vibrations for amide and urea groups were observed in the range of 3154 to $3290 \mathrm{~cm}^{-1}$. The vibrations for urea carbonyls were found at 1705 to $1720 \mathrm{~cm}^{-1}$ and the amide carbonyls at 1688 to $1705 \mathrm{~cm}^{-1}$. The ${ }^{1} \mathrm{H}$ NMR spectra display the typical ABCD pattern for the orthodisubstituted benzamide and the $\mathrm{A}_{2} \mathrm{~B}_{2}$ pattern for the nitrophenyl group. Both urea hydrogens are shifted downfield in the range of 10.40 to $10.62 \mathrm{ppm}$ and the amide hydrogen at 8.70 to $8.85 \mathrm{ppm}$. The signals for the aliphatic hydrogens appear upfield (Fig. S1-S6†).

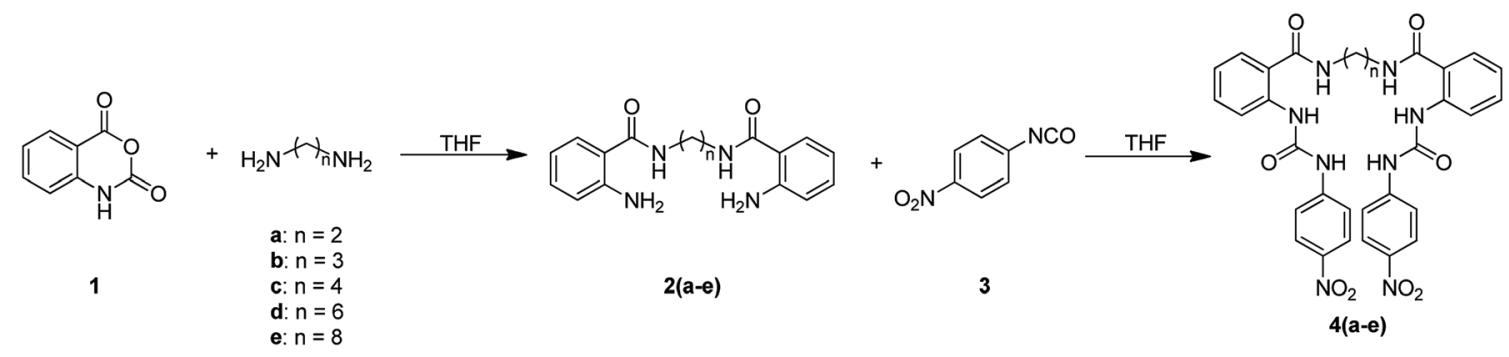

Scheme 1 
<smiles>CC(=O)c1cccc([N+](=O)[O-])c1</smiles><smiles>N=C(I)C(N)CN</smiles>

a: $n=2$

b: $n=3$

c: $n=4$

d: $n=6$

e: $n=8$<smiles>O=C(Nc1ccccc1)Nc1ccc([N+](=O)[O-])cc1</smiles><smiles>C=CNc1cccc(C(=O)NNCCNC(=O)c2cccc(NC)c2)c1</smiles>

8(a-e)

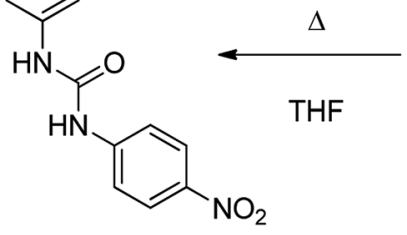<smiles>Nc1cccc(C=O)c1</smiles><smiles>Nc1cccc(C(=O)NC=NC2CCCCC2)c1</smiles><smiles>O=[N+]([O-])c1ccc([N+](=O)[O-])cc1</smiles>

$7(a-e)$

3

Scheme 2

For the synthesis of bis(ureas) 8 (meta receptors), 3-nitrobenzoyl chloride (5) was reacted with a dialkylamine in the presence of TEA at RT in anhydrous THF. The bis-(3-nitrobenzamide) intermediates 6 were obtained with 88\%, 90\%,
$80 \%, 59 \%$, and $25 \%$ yields, respectively. Then, the nitro groups were reduced to amino using an $\mathrm{H}_{2}$ atmosphere and $\mathrm{Pd}(10 \%) / \mathrm{C}$ at RT in dry $\mathrm{MeOH}$. The bis-(3-aminobenzamide) intermediates 7 were obtained with $42 \%, 98 \%, 69 \%, 80 \%$, and $90 \%$ yields. a)

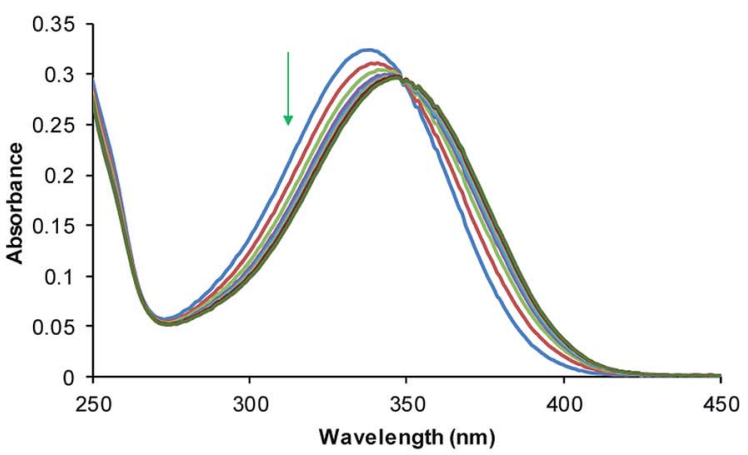

c)

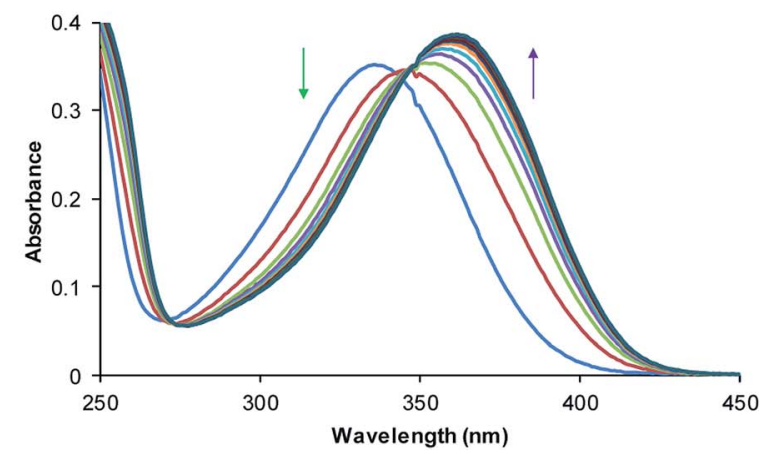

b)

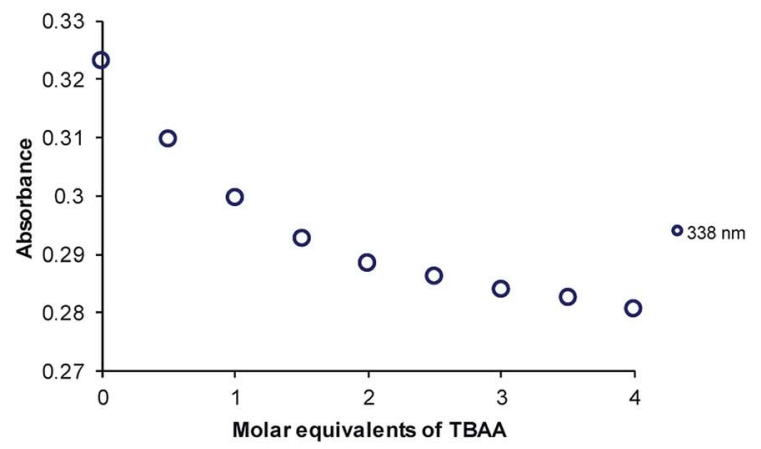

d)

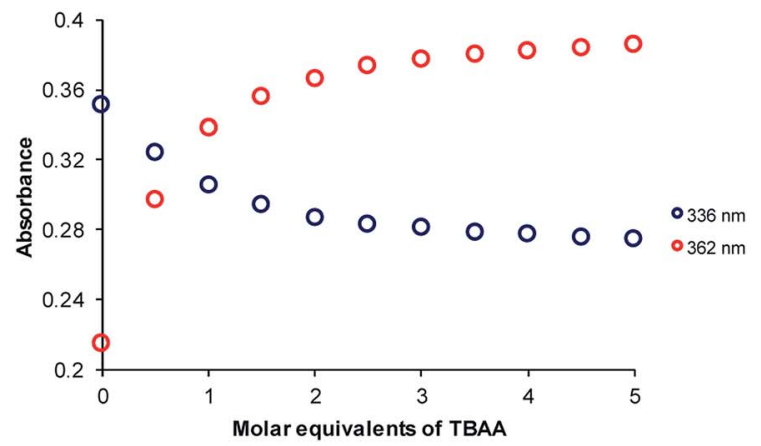

Fig. 2 UV-vis spectra and absorbance profiles obtained in the titration of $4 \mathrm{~d}$ ( $\mathrm{a}$ and $\mathrm{b}$ ) and $8 \mathrm{~d}$ (c and d) with TBAA in acetonitrile [4d] and [8d]=1 $\times 10^{-5} \mathrm{M}$. 
Compounds 7 were reacted with 4-nitrophenyl isocyanate in THF to obtain the bis-urea receptors 8 in $52 \%, 77 \%, 71 \%$, and $97 \%$ yields (Scheme 2). A meta mono-ureylbenzamide was synthetized under the same experimental conditions using propylamine in a $20 \%$ global yield.

In the FTIR spectra, stretching $\mathrm{N}-\mathrm{H}$ vibrations for amide and urea groups were observed in the range of 3221 to $3331 \mathrm{~cm}^{-1}$. The vibrations for urea carbonyls were found in the range 1706$1714 \mathrm{~cm}^{-1}$ and the amide carbonyls in the range 1625$1640 \mathrm{~cm}^{-1}$. In the ${ }^{1} \mathrm{H}$ NMR spectra, the typical ABCX pattern for the meta-disubstituted benzamide and the $\mathrm{A}_{2} \mathrm{~B}_{2}$ pattern for the nitrophenyl group were observed. Both urea hydrogens were shifted downfield in the range of 9.05 to $9.47 \mathrm{ppm}$ and the amide hydrogen at 8.41 to $8.49 \mathrm{ppm}$. The signals for the aliphatic hydrogens appeared upfield (Fig. S7-S11†).

The chemical shift of urea and amide hydrogens in receptors $\mathbf{4}$ and $\mathbf{8}$ was directly related with the relative position of both functional groups, which was the result of a combination of resonance and inductive effects. The effect of the two electronwithdrawing groups, i.e., the nitrophenyl and benzamide, over these hydrogens was dramatically enhanced when they were in

Table 1 Association constants $(\log K)^{a}$ for receptors 4 with TBAX salts determined by UV-vis in $\mathrm{CH}_{3} \mathrm{CN}$

\begin{tabular}{lllll}
\hline $\mathrm{X}$ & $\log K_{11}$ & $\log K_{12}$ & $\log K_{21}$ \\
\hline & $\mathrm{AcO}^{-}$ & & $10.11(2)$ \\
& $\mathrm{BzO}^{-}$ & & $10.92(2)$ \\
& $\mathrm{F}^{-}$ & $5.62(1)$ & & \\
& & $5.09(2)$ & & \\
& & &
\end{tabular}

$$
4
$$

$4 a$

$4 \mathbf{b}$

$4 c$

$4 d$

$4 e$

$\begin{array}{lll}\mathrm{AcO}^{-} & 6.51(2) & \\ \mathrm{BzO}^{-} & 5.322(4) & \\ \mathrm{F}^{-} & 6.64(6) & 12.87(5) \\ \mathrm{H}_{2} \mathrm{PO}_{4}{ }^{-} & 6.3(1) & 11.9(1) \\ \mathrm{HP}_{2} \mathrm{O}_{7}{ }^{3-} & 5.55(4) & \\ \mathrm{AcO}^{-} & 5.689(4) & \\ \mathrm{BzO}^{-} & 4.661(4) & \\ \mathrm{F}^{-} & 6.08(5) & 11.41(4) \\ \mathrm{H}_{2} \mathrm{PO}_{4}{ }^{-} & 5.2(1) & 10.64(6) \\ \mathrm{HP}_{2} \mathrm{O}_{7}{ }^{3-} & 4.86(6) & \\ \mathrm{AcO}^{-} & 6.012(8) & \\ \mathrm{BzO}^{-} & 4.941(2) & \\ \mathrm{F}^{-} & 6.34(7) & 11.98(7) \\ \mathrm{H}_{2} \mathrm{PO}_{4}{ }^{-} & 7.54(6) & 12.50(8) \\ \mathrm{HP}_{2} \mathrm{O}_{7}{ }^{3-} & 5.73(5) & \\ \mathrm{AcO}^{-} & 5.321(2) & \\ \mathrm{BzO}^{-} & 4.806(3) & \\ \mathrm{F}^{-} & 6.07(4) & 11.50(3) \\ \mathrm{H}_{2} \mathrm{PO}_{4}^{-} & 6.55(2) & \\ \mathrm{HP}_{2} \mathrm{O}_{7}{ }^{3-} & 7.4(4) & \\ \mathrm{AcO}^{-} & 4.997(3) & \\ \mathrm{BzO}^{-} & 4.716(3) & \\ \mathrm{F}^{-} & & 9.14(2) \\ \mathrm{H}_{2} \mathrm{PO}_{4}{ }^{-} & 6.85(2) & 12.24(7) \\ \mathrm{HP}_{2} \mathrm{O}_{7}{ }^{3-} & 6.9(1) & \\ & \end{array}$

${ }^{a}$ Standard deviation of the fit is given in parenthesis. the ortho position. The acidity and the spatial availability of these hydrogens depends on the position and may result in different interaction strength with anions.

\section{Study of anion recognition by UV-vis}

The ortho and meta $N$-propyl ureabenzamides and receptors 4 and 8 presented an absorption band at 330-340 $\mathrm{nm}$ due to the nitrophenyl chromophore. The changes in this band were monitored by titration with different TBAX salts $\left(\mathrm{X}=\mathrm{AcO}^{-}(\mathrm{A})\right.$, $\mathrm{BzO}^{-}$(B), $\mathrm{F}^{-}$(F), $\mathrm{H}_{2} \mathrm{PO}_{4}^{-}$(HP), and $\mathrm{HP}_{2} \mathrm{O}_{7}{ }^{3-}$ (HPP)) in acetonitrile. All the receptors showed spectral changes in the titration depending on the receptor structure and the anion. Fig. 2 shows the spectra obtained in the titration of receptors $4 \mathbf{d}$ and $8 \mathbf{d}$ with TBAA in acetonitrile. There were bathochromic and hypochromic shifts in the absorption of receptor $4 \mathbf{d}$ at $338 \mathrm{~nm}$ during the titration, indicating the interaction with the anion, while there were bathochromic and hyperchromic shifts in the absorption band at $336 \mathrm{~nm}$ in receptor 8d. A new maximum absorbance band was observed at $362 \mathrm{~nm}$. As can be seen, the changes in $\mathbf{8 d}$ were more evident than in $\mathbf{4 d}$. In general, there

Table 2 Association constants $(\log K)^{a}$ for receptors 8 with TBAX salts determined by UV-vis in $\mathrm{CH}_{3} \mathrm{CN}$

\begin{tabular}{lllll}
\hline Receptor & $\mathrm{X}$ & $\log K_{11}$ & $\log K_{12}$ & $\log K_{21}$ \\
\hline & $\mathrm{AcO}^{-}$ & $7.5(2)$ & & $13.7(4)$ \\
& $\mathrm{BzO}^{-}$ & $5.931(4)$ & & \\
$\mathrm{F}^{-}$ & $8.1(2)$ & & $13.5(2)$ \\
& $\mathrm{H}_{2} \mathrm{PO}_{4}^{-}$ & $6.31(1)$ & $12.1(1)$ & \\
& $\mathrm{HP}_{2} \mathrm{O}_{7}{ }^{3-}$ & $6.31(1)$ & & \\
& & & &
\end{tabular}

$8 \mathbf{a}$

$10.75(7)$

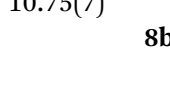

$10.38(5)$

8c

$11.27(9)$

$8 d$

$13.9(9)$

8 e

$13.3(3)$

\begin{tabular}{|c|c|c|c|}
\hline $\mathrm{AcO}^{-}$ & $6.84(2)$ & & \multirow[b]{5}{*}{$10.93(2)$} \\
\hline $\mathrm{BzO}^{-}$ & $6.007(6)$ & & \\
\hline $\mathrm{F}^{-}$ & $5.050(4)$ & & \\
\hline $\mathrm{H}_{2} \mathrm{PO}_{4}^{-}$ & $5.76(5)$ & $11.17(4)$ & \\
\hline $\mathrm{HP}_{2} \mathrm{O}_{7}^{3-}$ & & & \\
\hline $\mathrm{AcO}^{-}$ & $5.90(5)$ & $10.0(2)$ & \multirow{7}{*}{$10.94(1)$} \\
\hline $\mathrm{BzO}^{-}$ & $5.83(4)$ & $9.83(9)$ & \\
\hline $\mathrm{F}^{-}$ & $8.4(3)$ & $13.9(3)$ & \\
\hline $\mathrm{H}_{2} \mathrm{PO}_{4}^{-}$ & $5.932(8)$ & $11.29(1)$ & \\
\hline $\mathrm{HP}_{2} \mathrm{O}_{7}^{3-}$ & & & \\
\hline $\mathrm{AcO}^{-}$ & $5.502(6)$ & & \\
\hline $\mathrm{BzO}^{-}$ & $5.160(5)$ & & \\
\hline $\mathrm{F}^{-}$ & $8.6(3)$ & $14.7(3)$ & \multirow{5}{*}{$14.3(1)$} \\
\hline $\mathrm{H}_{2} \mathrm{PO}_{4}^{-}$ & $5.12(3)$ & $10.51(3)$ & \\
\hline $\mathrm{HP}_{2} \mathrm{O}_{7}^{3-}$ & $7.02(7)$ & & \\
\hline $\mathrm{AcO}^{-}$ & $6.01(5)$ & $10.0(2)$ & \\
\hline $\mathrm{BzO}^{-}$ & $5.80(3)$ & $10.25(5)$ & \\
\hline $\mathrm{F}^{-}$ & $5.20(2)$ & & \multirow{5}{*}{$14.5(1)$} \\
\hline $\mathrm{H}_{2} \mathrm{PO}_{4}^{-}$ & $7.74(7)$ & $13.43(7)$ & \\
\hline $\mathrm{HP}_{2} \mathrm{O}_{7}^{3-}$ & $6.80(9)$ & & \\
\hline $\mathrm{AcO}^{-}$ & $5.89(6)$ & $9.8(5)$ & \\
\hline $\mathrm{BzO}^{-}$ & $5.55(5)$ & $9.2(4)$ & \\
\hline \multicolumn{4}{|l|}{$\mathrm{F}^{-}$} \\
\hline $\mathrm{H}_{2} \mathrm{PO}_{4}^{-}$ & $6.07(3)$ & $11.88(4)$ & \\
\hline $\mathrm{HP}_{2} \mathrm{O}_{7}^{3-}$ & & & $11.90(3)$ \\
\hline
\end{tabular}

${ }^{a}$ Standard deviation of the fit is given in parenthesis. 


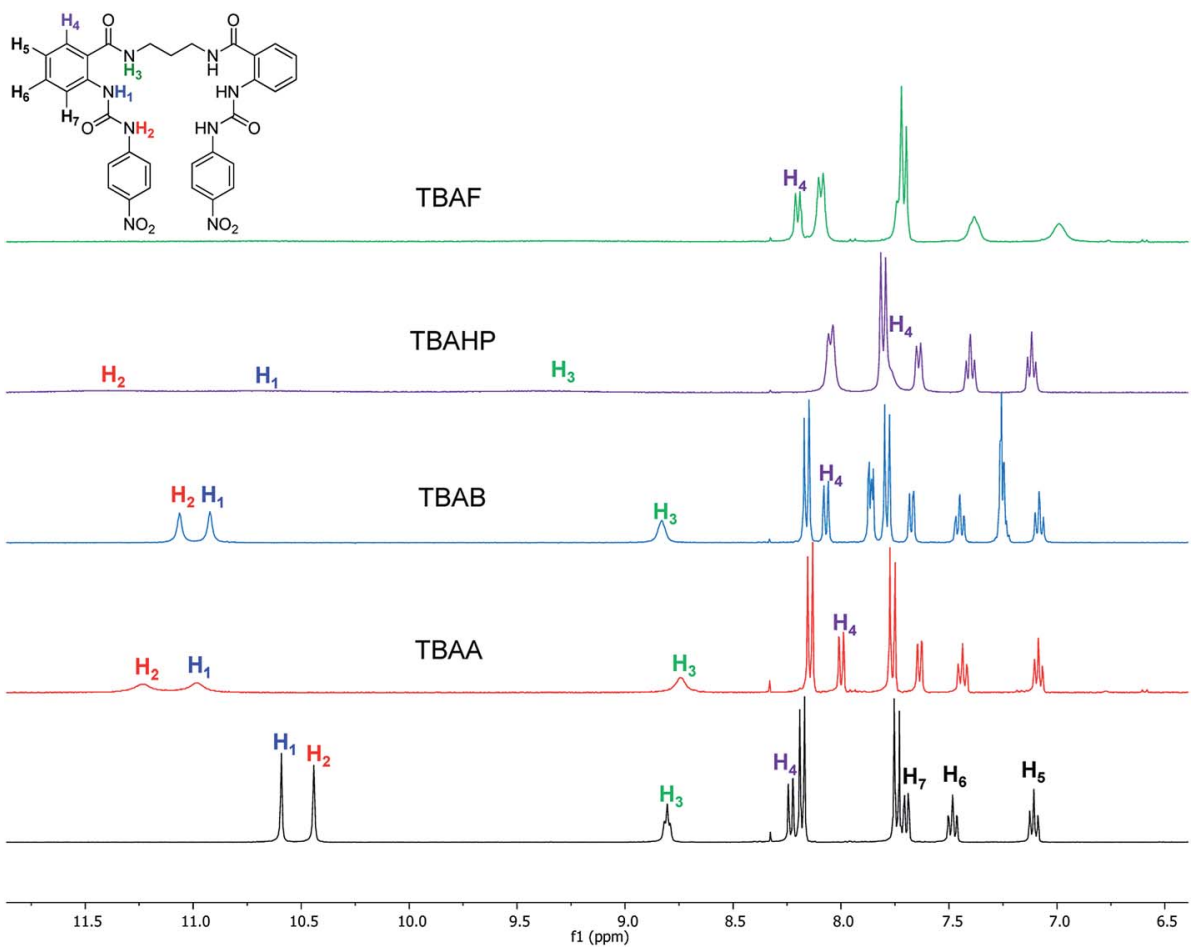

Fig. 3 Partial ${ }^{1} \mathrm{H}-\mathrm{NMR}$ spectra of free $4 \mathrm{~b}$ and with two molar equivalents of TBAA, TBAB, TBAHP, and TBAF in DMSO- $d_{6}$.

were more evident spectroscopic changes in receptors 8 than in receptors 4 , which was directly related to the relative position of the urea and benzamide groups in these receptors (Fig. S12S23 $\dagger$ ). All the receptors showed a spectroscopic response toward the studied anions. However, the TBHPP induced the greatest changes in the absorption bands when lower molar equivalents were added. This was probably due to the higher number negative charges and the geometry of this anion. The TBAHPP salt induced a partial deprotonation at the ortho $N$-propyl ureabenzamide, which was evidenced by the presence of a band at $450 \mathrm{~nm}$ (Fig. S12i†). However, this new band was not observed

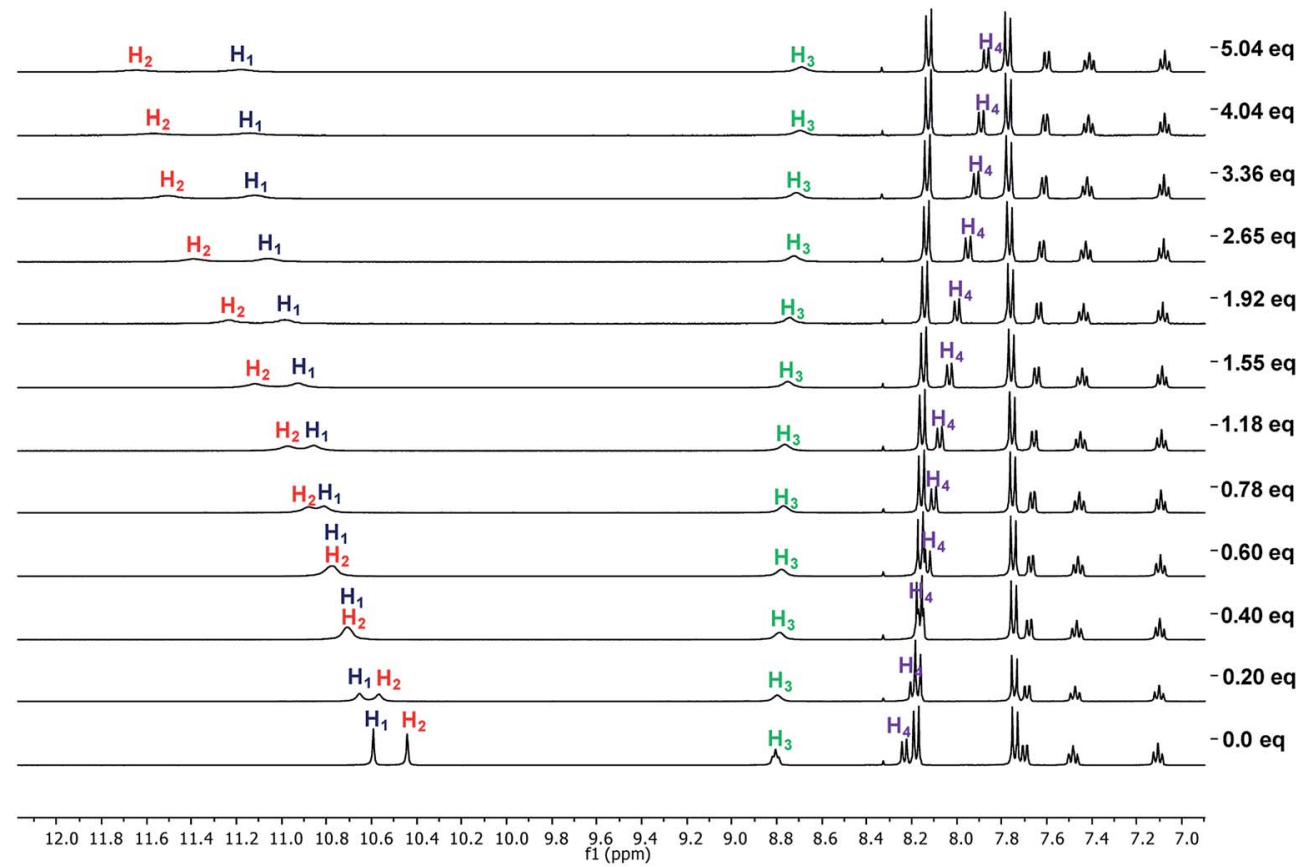

Fig. 4 Partial ${ }^{1} \mathrm{H}-\mathrm{NMR}$ spectra obtained in the titration of $4 \mathrm{~b}(6 \mathrm{mM})$ with TBAA in DMSO- $d_{6}$. 
Table 3 Association constants $(\log K)^{a}$ for the complexes of receptors 4 with different TBAX salts determined by ${ }^{1} \mathrm{H}-\mathrm{NMR}$

\begin{tabular}{llll}
\hline Receptor & $\mathrm{X}$ & $\log K_{11}$ & $\log K_{12}$ \\
\hline 4b & $\mathrm{AcO}^{-}$ & $1.902(9)$ & \\
& $\mathrm{BzO}^{-}$ & $1.701(7)$ & \\
& $\mathrm{H}_{2} \mathrm{PO}_{4}{ }^{-}$ & $2.23(1)$ & \\
& & & \\
& & & \\
& & $2.09(2)$ & \\
& $\mathrm{AcO}^{-}$ & $1.99(4)$ & \\
& $\mathrm{BzO}^{-}$ & 3.1 & \\
& $\mathrm{H}_{2} \mathrm{PO}_{4}{ }^{-b}$ & $2.02(2)$ & \\
& $\mathrm{AcO}^{-}$ & $2.11(1)$ & \\
& $\mathrm{BzO}^{-}$ & 2.5 & \\
& $\mathrm{H}_{2} \mathrm{PO}_{4}{ }^{-b}$ & $1.92(4)$ & \\
& $\mathrm{AcO}^{-}$ & $1.85(2)$ & \\
$\mathrm{BzO}^{-}$ & 2.3 &
\end{tabular}

${ }^{a}$ Standard deviation of the fit is given in parenthesis. ${ }^{b}$ Excessive standard deviation.

in the titrations of meta $N$-propyl ureabenzamide and receptors 4 and 8.

Table 1 lists the association constants $(\log K)$ for the complexes of receptors 4 calculated from the absorbance data using the HypSpec program, ${ }^{21-23}$ and are compared with those obtained with the ortho mono-ureylbenzamide receptor. Interestingly, the $N$-propyl mono-ureylbenzamide formed complexes with a $2: 1$ (receptor : anion) stoichiometry with TBAA, TBAB, and TBAHPP, which agreed with the possible cooperativity of both ureylbenzamide units in receptors 4 with the shorter oligomethylene spacer chains, and $1: 1$ complexes with TBAF and TBAHP. This behavior was not observed with the previously reported $N$-benzyl ortho mono-ureylbenzamide, ${ }^{20}$ where all the complexes had a 1 : 1 stoichiometry and the $\log K$ values ranged from 3.85 to 4.35 . Thus, the stoichiometry and affinity toward the anions were improved with the $N$-propyl substituent. All receptors 4 formed exclusively a $1: 1$ complex with TBAA and TBAB and there was a tendency for the $\log K_{11}$ to decrease as the oligomethylene chain length increased. This behavior suggests the cooperativity of both ureylbenzamide units interacting with the carboxylate unit in these complexes. Two complexes with $1: 1$ and $1: 2$ (receptor : anion) stoichiometry were observed with TBAF and TBAHP; in particular, receptor 4e formed only the $1: 2$ complex. Noteworthy, two complexes with $2: 1$ and $1: 1$ stoichiometry were detected with TBAHPP and the values of $\log K_{11}$ and $\log K_{21}$ increased as the spacer length increased. This unusual behavior with pyrophosphate was due to its differences in size, shape, and charge. Also, the $\log K_{11}$ values for fluoride and phosphate complexes with receptors 4 were one or two units higher compared to the mono ureylbenzamide, which may indicate a cooperative effect of both interaction sites.

Table 2 lists the association constants $(\log K)$ for the complexes of receptors $\mathbf{8}$ calculated from the absorbance data using the HypSpec program ${ }^{\mathbf{2 1 - 2 3}}$ and these are compared with those obtained with the corresponding meta monoureylbenzamide. The mono-ureylbenzamide mainly formed

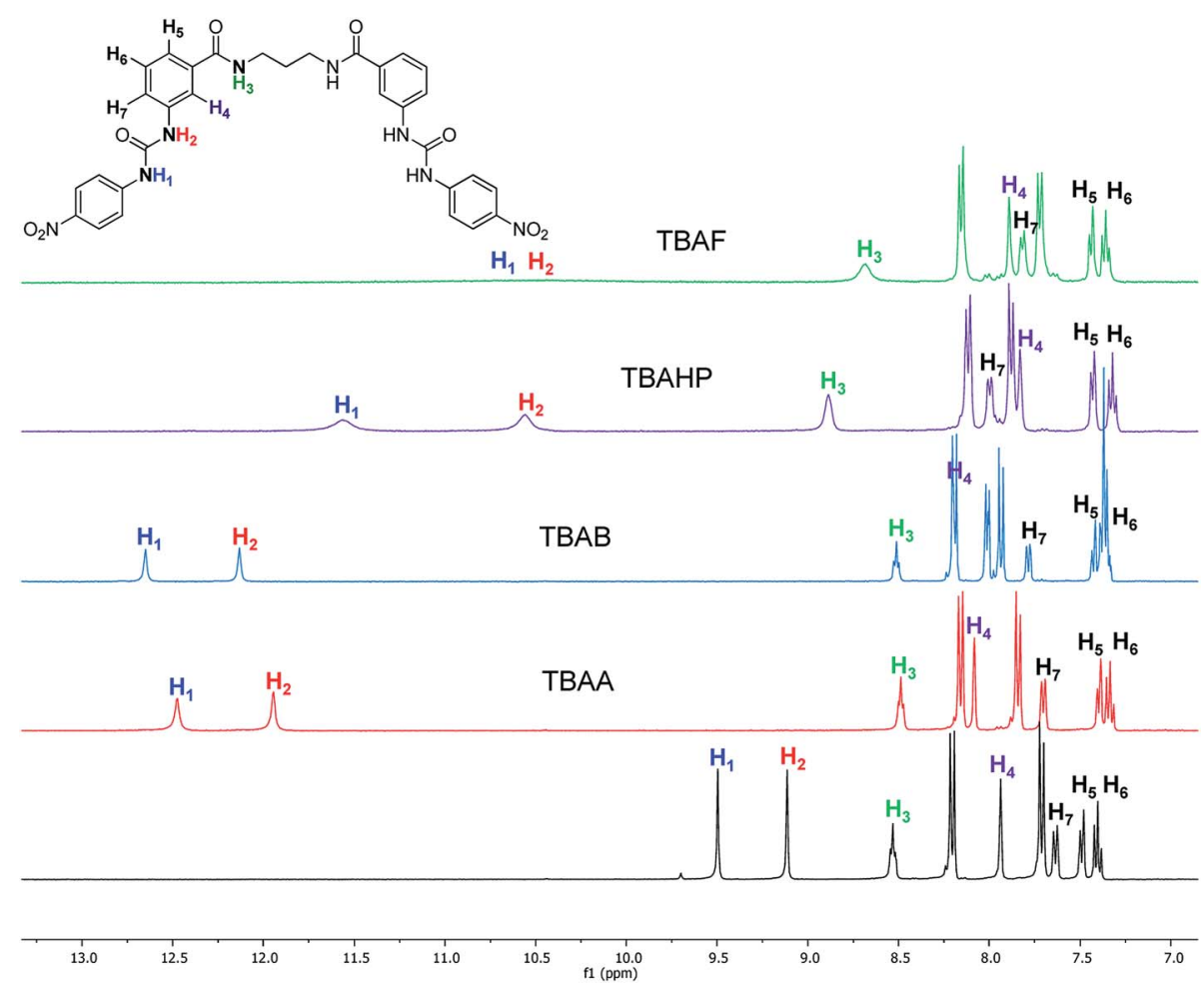

Fig. 5 Partial ${ }^{1} \mathrm{H}-\mathrm{NMR}$ spectra of free $8 \mathrm{~b}$ and with two molar equivalents of TBAA, TBAB, TBAHP, and TBAF in DMSO- $d_{6}$. 


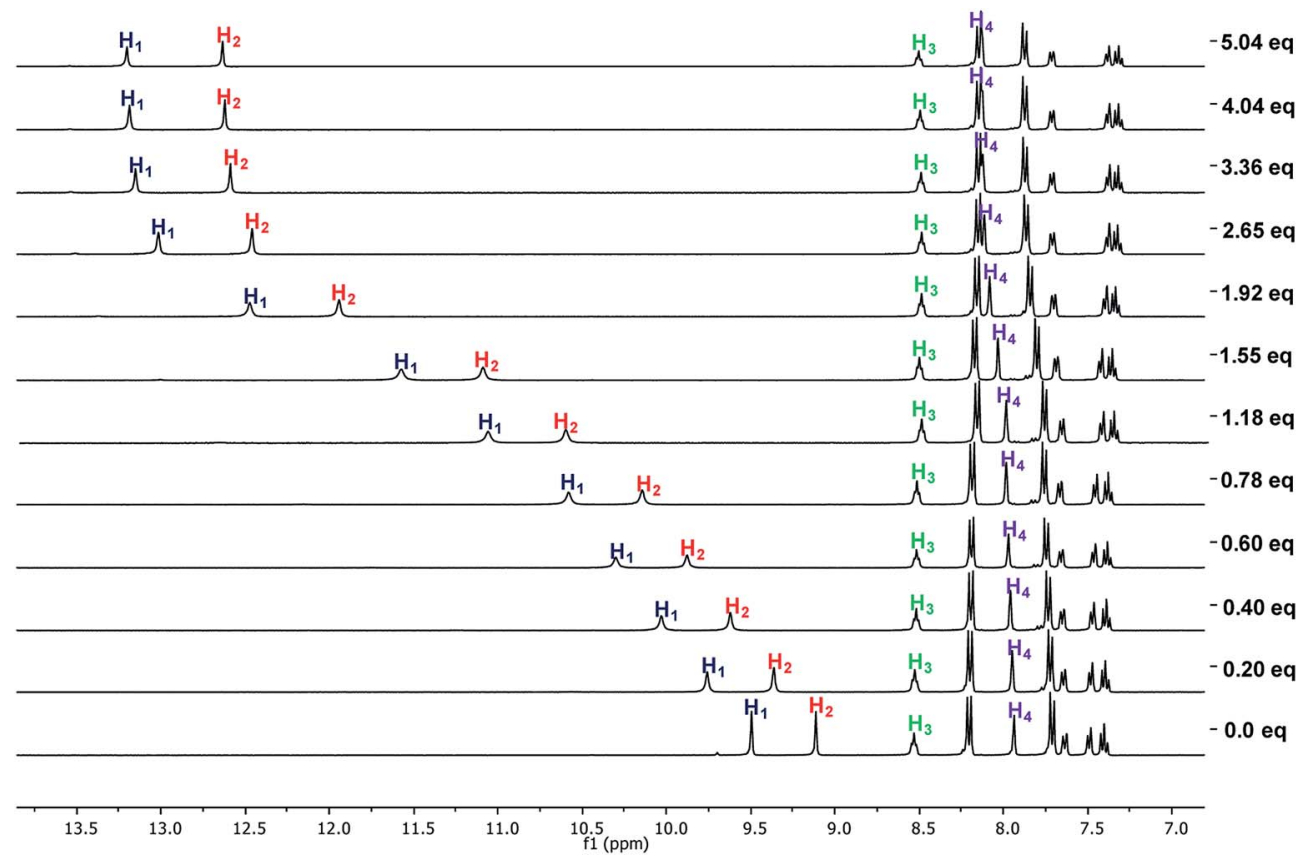

Fig. $6{ }^{1} \mathrm{H}$ NMR spectra obtained in the titration of $8 \mathrm{~b}$ with TBAA in DMSO.

complexes of a $1: 1$ receptor-anion stoichiometry with all the TBAX salts, and formed $2: 1$ complexes with acetate and fluoride, indicating a different behavior with its ortho analogue. The $\log K$ values were one to three units higher than those obtained with the $N$-benzyl ortho mono-ureylbenzamide previously reported ${ }^{20}$ evidencing the positive effect of the $\mathrm{N}$-propyl substituent in the affinity toward anions. Also, there were difference in the complex stoichiometries with TBAA and TBAF. In general, receptors 8 formed complexes with both $1: 1$ and $1: 2$ stoichiometries with the TBAX salts, and a $2: 1$ complex with TBAHPP. This anion may serve as a template for the self-assembly of two receptor molecules at low concentration, and there was a tendency for $\log K_{21}$ to increase as the oligomethylene chain length increased, indicating a higher affinity. The receptor 8a (with the shorter spacer chain) formed a $1: 1$ complex with

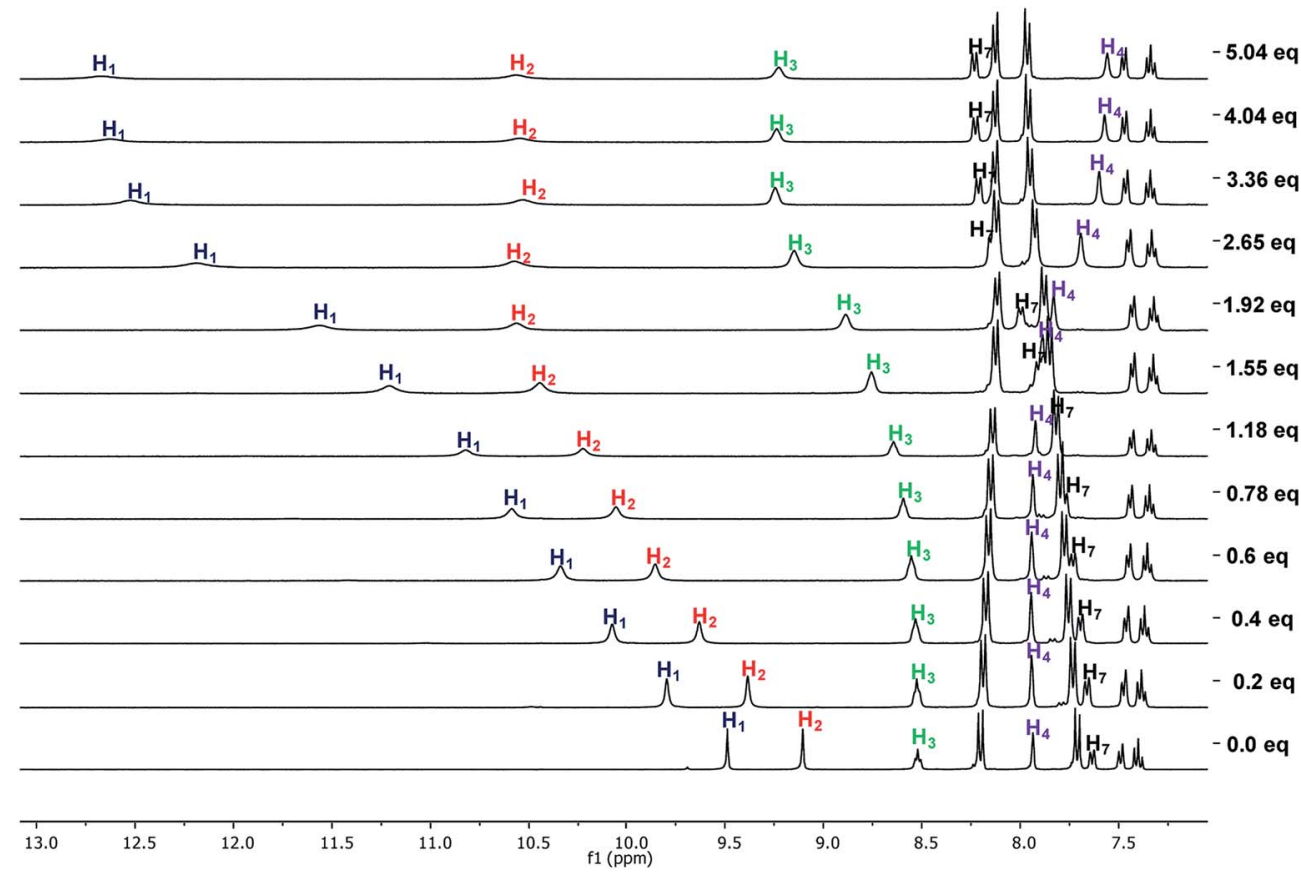

Fig. $7{ }^{1} \mathrm{H}$ NMR spectra obtained in the titration of $8 \mathrm{~b}$ with TBAHP in DMSO- $d_{6}$. 
TBAA, TBAB, and TBAF and a $1: 2$ complex with TBAHP, while $8 \mathbf{c}$ did this with TBAA and TBAB.

\section{Study of anion recognition by ${ }^{1} \mathrm{H}$ NMR}

The interaction of the ortho monorureylbenzamide, $\mathbf{4 b}, \mathbf{4 c}$, and 4d with TBAA, TBAB, TBAF, TBAHP, and TBAHPP was analyzed by ${ }^{1} \mathrm{H}$ NMR in DMSO- $d_{6}$ due to the solubility of these receptors (Fig. S24-S43†). As depicted in Fig. 3, all the anions promoted different changes in the NMR signals of receptor $\mathbf{4 b}$ once the complex was formed in solution because of their differences in size, shape, and basicity. There was a non-equivalent downfield shift of the urea signals with TBAA and TBAB, indicating that both hydrogens $\mathrm{H}_{1}$ and $\mathrm{H}_{2}$ interacted with the anion, but not with the same strength. The spectra obtained during the titration (Fig. 4) indicated that $\mathrm{H}_{2}$ was more affected than $\mathrm{H}_{1}$. Also, it is interesting that the amide hydrogen $\left(\mathrm{H}_{3}\right)$ signal was broadened, which means this hydrogen was also involved in the complex, and the $\mathrm{H}_{4}$ hydrogen at the ortho position to the amide carbonyl shifted to upfield when the interaction with the anion occurred. The same spectral changes were observed in the other the receptors with these anions.

There was a downfield shift and broadening of the $\mathrm{H}_{1}, \mathrm{H}_{2}$, and $\mathrm{H}_{3}$ signals, indicating a strong participation of the three hydrogens in the supramolecular complex with TBAHP. Again, $\mathrm{H}_{2}$ was more affected than $\mathrm{H}_{1}$ and $\mathrm{H}_{4}$, being considerably shifted upfield, probably due to a conformational change in the amide group, where the "ortho effect" over $\mathrm{H}_{4}$ was avoided. In the titration with TBAF, all the $\mathrm{NH}$ hydrogens disappeared, indicating an acid-base reaction; a change of color also occurred in the solution. The titration with TBAHPP showed the deprotonation of urea hydrogens since the first anion aliquot due to an acid-base reaction. Also, the signal of the amide hydrogens $\mathrm{H}_{3}$ underwent a downfield shift and widening, indicating a strong interaction with this hydrogen. The signal for $\mathrm{H}_{4}$ was considerably shifted to upfield and became broad. Noteworthy, there were exceptional changes in the NMR spectra when more than two equivalents of TBAHPP were added to the solution. The signals of the $\mathrm{A}_{2} \mathrm{~B}_{2}$ system for the nitrophenyl group were collapsed in one signal, and the rest of the signals showed significant changes in their chemical shifts and become broad. The same patterns were observed with the rest of the studied receptors (Fig. S28, S33, S38 and S43†).

The association constants for the complexes of ortho monoureylbenzamide, $\mathbf{4 b}, \mathbf{4 c}$, and $\mathbf{4 d}$ receptors were calculated from the chemical shifts data using the HypNMR program ${ }^{21-23}$ and are reported in Table 3. As expected, the ortho monoureylbenzamide receptor formed complexes with a $1: 1$ stoichiometry with all the studied anions. The chemical shift profiles obtained with TBAA and TBAB fitted the $1: 1$ stoichiometry model, and the values of $\log K_{11}$ decreased as the oligomethylene chain length increased in receptors 4 . These association constants are comparable to those reported in the literature for other receptors, ${ }^{19}$ even though receptors 4 had a flexible interconnection chain. The chemical shift profiles in the titration with TBAHP fitted $1: 1$ and $1: 2$ stoichiometries and the values of $\log K_{11}$ were higher than those obtained with
TBAA and TBAB. Both $\log K_{11}$ and $\log K_{12}$ values depended on the oligomethylene chain length decreasing as the oligomethylene chain increased. The values of $\log K$ with TBAF and TBAHPP could not be calculated due to the deprotonation reaction. The stoichiometry of the supramolecular complexes was in agreement with those found by UV-vis for these receptors.

The interaction of the receptors meta mono-ureylbenzamide, $\mathbf{8 b}, \mathbf{8 c}$, and $\mathbf{8 d}$ with TBAA, TBAB, TBAF, TBAHP, and TBAHPP was analyzed by ${ }^{1} \mathrm{H}$ NMR in DMSO- $d_{6}$ (Fig. S44-S63†). As seen in Fig. 5, all the TBAX salts promoted different changes in the signals of receptor $\mathbf{8 b}$ once the complex was formed in solution. Here, an equivalent downfield shift of the urea signals with TBAA and TBAB was observed, indicating that both hydrogens interacted with the anion with the same strength. The spectra obtained during the titration with TBAA (Fig. 6) indicated that both $\mathrm{H}_{2}$ and $\mathrm{H}_{1}$ were gradually shifted downfield when up to two equivalents of TBAA were added. The amide hydrogen $\left(\mathrm{H}_{3}\right)$ slightly shifted to upfield and interestingly, the hydrogen at the amide carbonyl and urea ortho position $\left(\mathrm{H}_{4}\right)$ shifted to downfield, suggesting a bond polarization due to a $\mathrm{C}-\mathrm{H} \cdots \mathrm{O}$ interaction in the complex. This effect over the $\mathrm{H}_{4}$ signal was stronger with TBAB than with TBAA, and it was partially lost by increasing the oligomethylene chain length in receptors $8 \mathbf{c}$ and $8 d$.

The successive formation of three different complexes was detected in the titration of $\mathbf{8 b}$ with TBAHP (Fig. 7). The two urea signals were shifted downfield with the same magnitude when one equivalent of TBAHP was added. Then, the chemical shift of $\mathrm{H}_{1}$ was higher than $\mathrm{H}_{2}$ when from one to three equivalents of anion was added, but beyond this concentration it remained constant. The signal of $\mathrm{H}_{2}$ was gradually shifted downfield when up to two equivalents of anion were added. The amide signal became wide when one equivalent of anion was added, and was then shifted downfield with the addition from one to three

Table 4 Association constants $(\log K)^{a}$ for the complexes of receptor 8 with different TBAX salts determined by ${ }^{1} \mathrm{H}-\mathrm{NMR}$ in DMSO- $d_{6}$

\begin{tabular}{lllll}
\hline $\mathrm{X}$ & $\log K_{11}$ & $\log K_{12}$ & $\log K_{13}$ \\
\hline & $\mathrm{AcO}^{-}$ & $3.61(6)$ & & \\
& $\mathrm{BzO}^{-}$ & $3.25(3)$ & & \\
& $\mathrm{H}_{2} \mathrm{PO}_{4}{ }^{-b}$ & 5.0 & 8.2 & \\
& & & \\
& & & \\
& & & & \\
& $\mathrm{AcO}^{-}$ & $4.11(1)$ & $7.6(2)$ & 12.6 \\
& $\mathrm{BzO}^{-}$ & $5.3(4)$ & $8.9(7)$ & \\
& $\mathrm{H}_{2} \mathrm{PO}_{4}{ }^{-b}$ & 5.1 & 9.4 & \\
& $\mathrm{AcO}^{-}$ & & $5.46(8)$ & 11.3 \\
& $\mathrm{BzO}^{-}$ & & $5.33(1)$ & \\
& $\mathrm{H}_{2} \mathrm{PO}_{4}{ }^{-b}$ & 4.9 & 9.0 & \\
& $\mathrm{AcO}^{-}$ & & $5.29(1)$ & \\
& $\mathrm{BzO}^{-}$ & $3.0(6)$ & $5.91(9)$ & \\
& $\mathrm{H}_{2} \mathrm{PO}_{4}{ }^{-b}$ & 2.50 & 4.47 &
\end{tabular}

${ }^{a}$ Standard deviation of the fit is given in parenthesis. ${ }^{b}$ Excessive standard deviation. 
a)

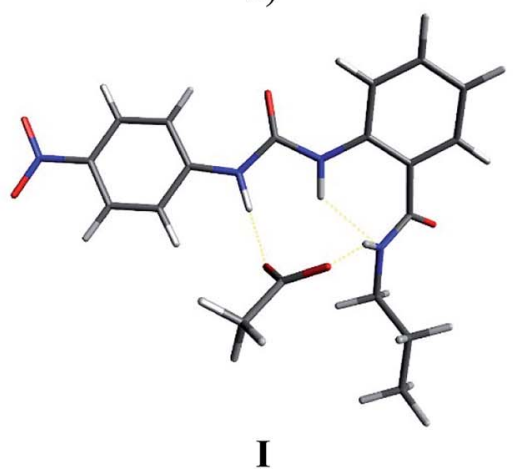

b)

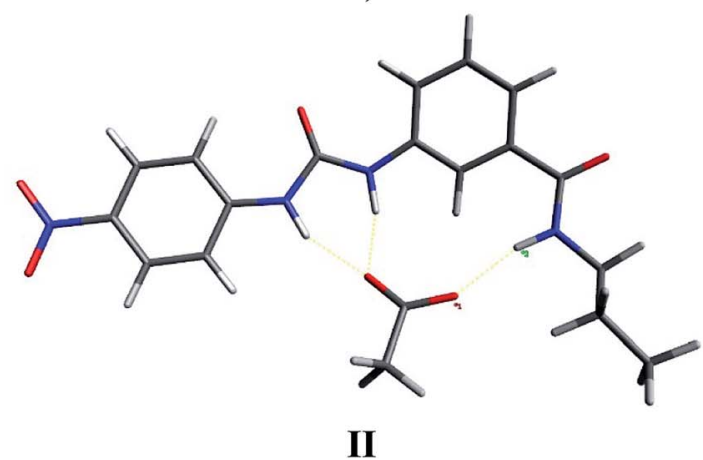

Fig. 8 Optimized structures of monoureabenzamide-TBAA complexes I (a) and II (b) by DFT.

equivalents and then remained constant. Interestingly, the signal of $\mathrm{H}_{4}$ was shifted to upfield and the signal of $\mathrm{H}_{7}$ was shifted to downfield, indicating a significant change in the chemical environment of these hydrogens. Considering the changes of the signals, the urea hydrogens as well as the amide hydrogen equally participated in the $1: 1$ complex (see Fig. S66 $\dagger$ ), while the hydrogens $\mathrm{H}_{1}$ and $\mathrm{H}_{3}$ were more affected in the $1: 2$ complex. The chemical shifts of $\mathrm{H}_{4}$ and $\mathrm{H}_{7}$ indicated a conformational change in the receptor, allowing the interaction with a third anion unit, where the $\mathrm{H}_{1}$ and $\mathrm{H}_{3}$ hydrogens were the most affected. Receptors $\mathbf{8 c}$ and $\mathbf{8 d}$ showed a similar signal pattern with TBAHP, although the $\mathrm{H}_{3}$ signal was less affected as the oligomethylene chain increased (Fig. S57 and S62†).

The titrations of $\mathbf{8 b}, \mathbf{8 c}$, and $\mathbf{8 d}$ with TBAHPP showed deprotonation of the urea groups since the first aliquot was added. Interestingly, all the signals become broad and shifted in different ways, indicating three clear stages during the course of the titration. All the signals showed the maximum broadening between 0.4 and 0.6 molar equivalents of TBAHPP added, suggesting a $2: 1$ (receptor : anion) complex. The same behavior was also found with the UV titrations.
Then, all the signals recovered their multiplicity when $\mathbf{1 . 2}$ equivalents were added, and the $\mathrm{H}_{3}$ signal was shifted $1.8 \mathrm{ppm}$ downfield, indicating the interaction with the anion. These changes showed the formation of a $1: 1$ complex. Interestingly, an additional signal shifted about $0.5 \mathrm{ppm}$ appeared for $\mathrm{H}_{3}$, and the two signal of the nitrophenyl group collapsed into one at 2.4 equivalents of TBAHPP added, showing changes in the complex. All these changes in the NMR spectra indicated an acid-base reaction, but also the interaction with the anion in different forms depending on the anion concentration.

The association constants $(\log K)$ for the complexes of receptors meta mono-ureylbenzamide, $\mathbf{8 b}, \mathbf{8 c}$, and $\mathbf{8 d}$ were calculated from the chemical shifts and are reported in Table 4 . The meta mono-ureylbenzamide formed $1: 1$ complexes with TBAA and TBAB salts, and the data fitting showed the presence of additional complexes with TBAHP. The chemical shift profiles obtained with TBAA and TBAB fitted with a $1: 1$ and $1: 2$ stoichiometry model, where the values of $\log K$ decreased as the oligomethylene chain length increased in receptors 8 . The values of $\log K$ were considerably superior compared to those obtained with receptors 4 . a)

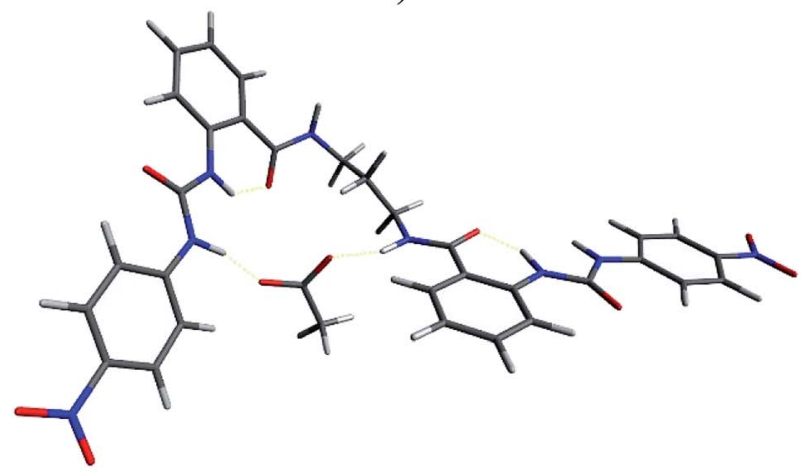

III

$\mathrm{E}_{\text {rel }}=9.76 \mathrm{kcal} / \mathrm{mol}$ b)

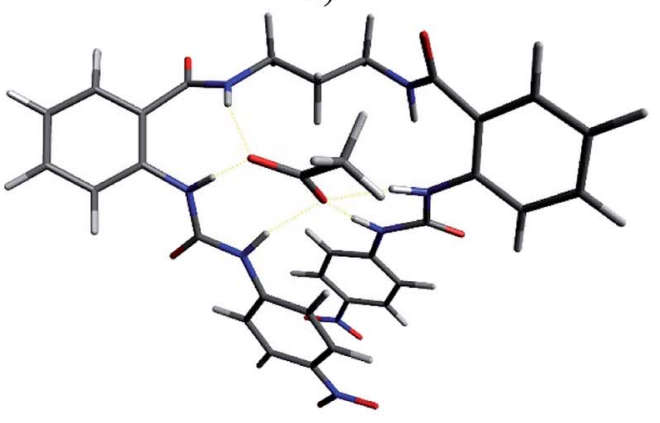

IV

$\mathrm{E}_{\text {rel }}=0 \mathrm{kcal} / \mathrm{mol}$

Fig. 9 Optimized structures III (a) and IV (b) for complex $4 \mathrm{~b}-\mathrm{AcO}^{-}$by DFT. 
a)

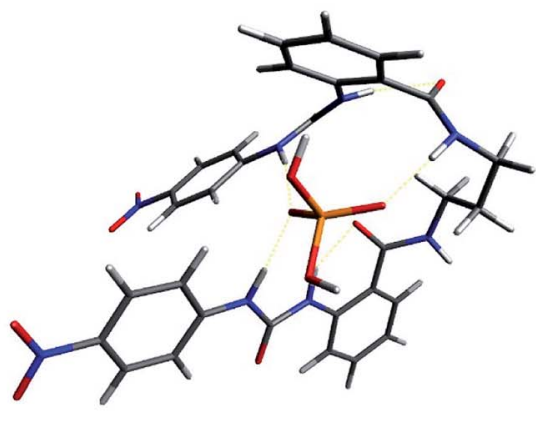

V b)

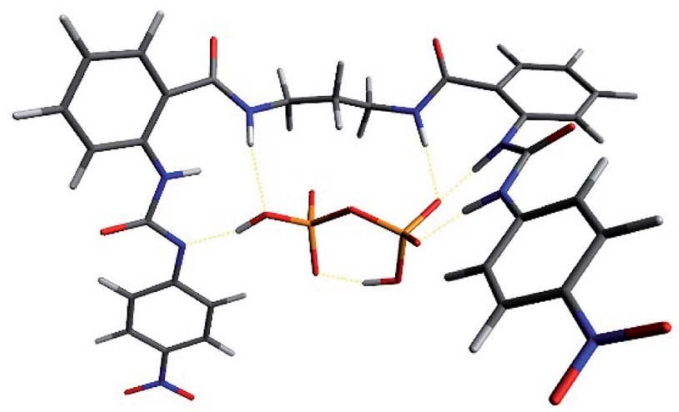

VI

Fig. 10 Optimized structures $\mathrm{V}(\mathrm{a})$ and $\mathrm{VI}(\mathrm{b})$ for the complexes $4 \mathrm{~b}-\mathrm{H}_{2} \mathrm{PO}_{4}{ }^{-}$and $4 \mathrm{~b}-\mathrm{HP}_{2} \mathrm{O}_{7}{ }^{3-}$ by DFT.

The results obtained by UV-vis and ${ }^{1} \mathrm{H}$ NMR demonstrated that receptors $\mathbf{4}$ and $\mathbf{8}$ established interactions with all the anions (TBAX salts) used in the study. However, there were significant differences regarding the urea and amide position, the oligomethylene chain length, and the geometry and charge of the anion. Receptors 8 showed more evident spectroscopic changes due to the interaction with the anion compared with the observed response of receptors 4 , which was attributed to the different position of the urea and amide groups in the receptors. The values of the association constants obtained by UV-vis were in the same orders for both types of receptors $\left(\log K_{11}=4-8, \log K_{12}=10-15, \log K_{21}=10-15\right)$. This similarity in the affinity was related to the strength of the interaction with the anions, considering that in receptors $\mathbf{4}$, three hydrogen bonding per each ureabenzamide unit may participate in the supramolecular complex, as was observed in the NMR analysis. The fitting of the absorbance data revealed the formation of $2: 1$ (receptor: anion) complexes with TBAA, TBAB, and TBAHPP with the ortho mono-ureylbenzamide. This behavior was consistent with the formation of $1: 1$ complexes with these anions in receptors $\mathbf{4}$, where the two ureylbenzamide units participated in the interaction with one unit of the anion. Also the increase of the association constants in the receptors with the shorter oligomethylene chains (4a and $4 \mathbf{b}$ ) confirmed a major cooperativity between the two units due to their proximity. Instead, there was an opposite tendency with TBAHPP in both $1: 1$ and $2: 1$ (receptor : anion) complexes, probably due to the different geometry and higher number of negative charges. The successive formation of $1: 1$ and $1: 2$ complexes with TBAF and TBAHP indicated a low stability of the $1: 1$ complexes, probably due to size and geometry of these anions compared with the carboxylates and pyrophosphate.

The successive formation of $1: 1$ and $1: 2$ with TBAA, TBAB, TBAF, and TBAHP, and only $2: 1$ complexes with TBAHPP at low concentration occurred in receptors $\mathbf{8}$, where this latter case is an interesting peculiarity. A significant difference in receptors $\mathbf{8}$ was the strong equivalent interaction of the urea hydrogens and the apparent $\mathrm{C}-\mathrm{H} \cdots \mathrm{O}$ interaction with carboxylates. Interestingly, the tetrahedral geometry of dihydrogen phosphate induces an interaction with the receptor with different
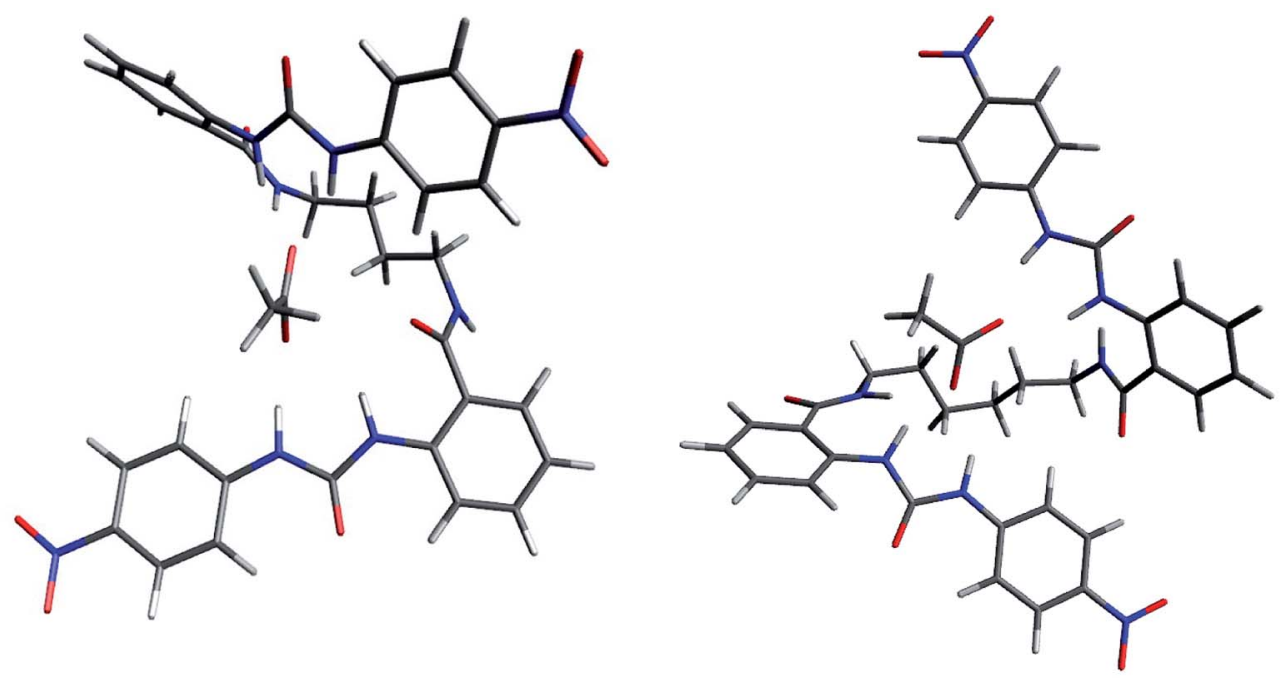

Fig. 11 Optimized structures for the complexes $4 c-\mathrm{AcO}^{-}$and $4 \mathrm{~d}-\mathrm{AcO}^{-}$by DFT. 
stoichiometries, where even the amide hydrogen is involved in the $1: 2$ and $1: 3$ complexes (receptor : anion). The formation of higher ratio complexes with TBAHP even with the meta $N$ propyl ureabenzamide up to $1: 3$ was rather unexpected. The fitting quality with this anion was also poor, but all attempts to exclude the higher ratio complexes failed; fitting to a simpler model always was divergent and did not allow any estimate of the binding constants. We believe that these complications resulted from the recently established strong auto-association of dihydrogen phosphate anion, particularly complicated by a possible contribution of free phosphoric acid, which may be generated by water traces in the solvent employed. ${ }^{25}$ Moreover, the UV-vis and NMR studies with TBAHPP demonstrated the formation of supramolecular complexes of different stoichiometries with all receptors at high concentration, which was consistent with the tendency observed in the UV-vis studies at low concentration.

\section{Theoretical calculations of the supramolecular complexes}

In order to support these experimental findings, the geometries of the supramolecular complexes were optimized by theoretical calculations using DFT with a B3LYP 6-31G(d) basis set. ${ }^{24}$ First, the mono-ureylbenzamide complexes with $\mathrm{AcO}^{-}$were calculated and interesting differences were observed (Fig. 8). The distance between the urea hydrogens $\mathrm{H}_{1}$ and $\mathrm{H}_{2}$ and the carboxylate was not equivalent, indicating a different strength in the ortho complex (structure I), and a strong interaction with amide hydrogen. The inequivalent chemical shift of the urea hydrogen signals and broadening of the amide hydrogen in the NMR spectra is now evident (Fig. S24 $\dagger$ ). Also, the amide carbonyl lay out of the plane with the aromatic ring, which is consistent with the upfield shift of $\mathrm{H}_{4}$ signal in the NMR spectra, indicating the loss of the "ortho effect" over this hydrogen by the carbonyl. In the meta complex (structure II), the $\mathrm{H}_{1}, \mathrm{H}_{2}, \mathrm{H}_{3}$, and $\mathrm{H}_{4}$ hydrogens were oriented toward the a)

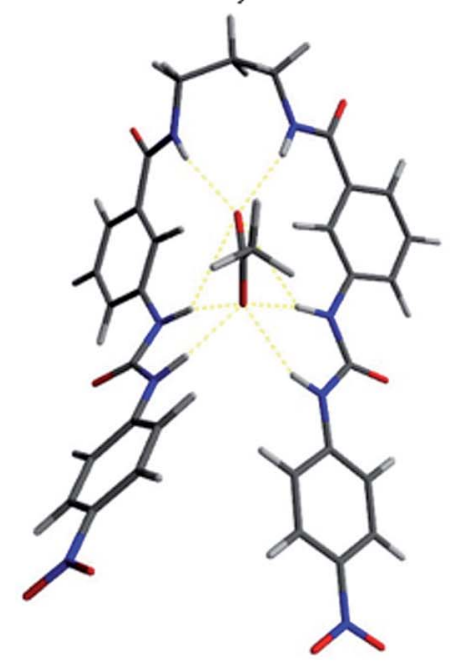

VII-Top view

c)

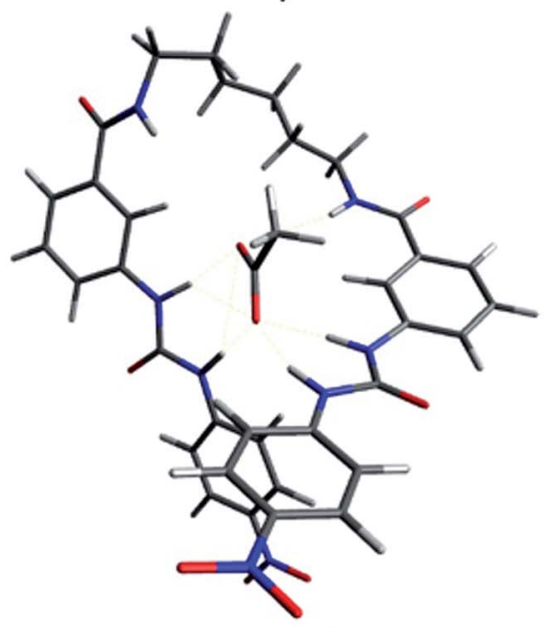

VIII-Top view b)

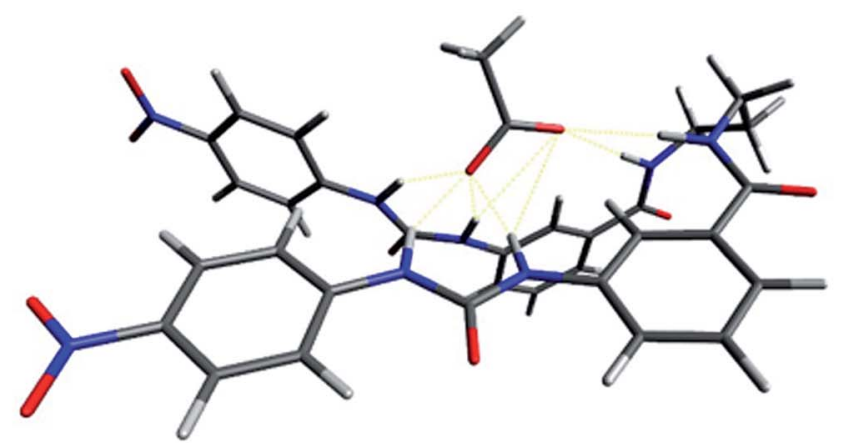

VII-Lateral view

d)

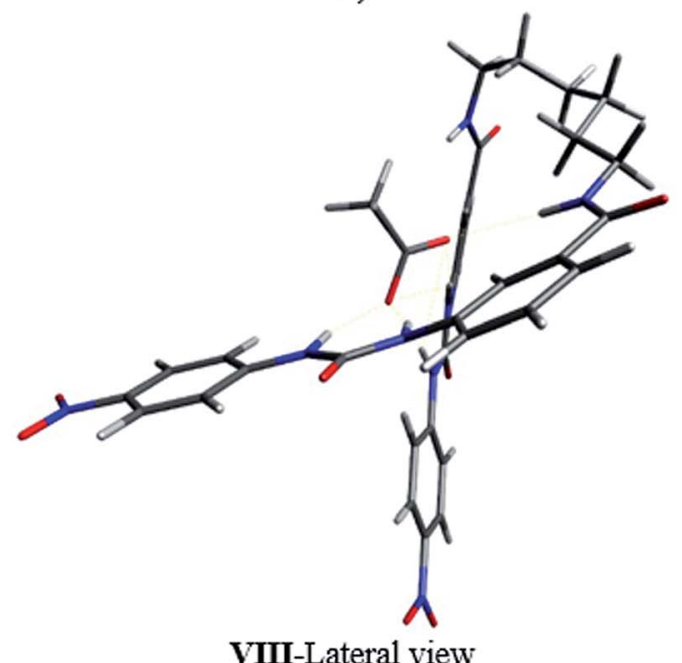

Fig. 12 Optimized structures for the complexes $8 \mathrm{~b}-\mathrm{AcO}^{-}(\mathrm{a}$ and $\mathrm{b})$ and $8 \mathrm{~d}-\mathrm{AcO}^{-}$(c and d) by DFT. 
carboxylate; the interaction of both urea hydrogens was equivalent and an interaction with amide hydrogen was present, but the distance between the carboxylate and the hydrogen was longer than that observed for the ortho complex. In this complex the distance of the $\mathrm{C}-\mathrm{H}_{4}$ with the carboxylate was about $2.2 \AA$, indicating a $\mathrm{C}-\mathrm{H} \cdots \mathrm{O}$ interaction which was consistent with the downfield of the $\mathrm{H}_{4}$ signal during the titration. The interactions in this model are in agreement with the results obtained in the NMR experiment (Fig. S44†).

Then, the interaction of $\mathbf{4} \mathbf{b}$ with $\mathrm{AcO}^{-}$was analyzed, optimizing a complex in which the $\mathrm{AcO}^{-}$interacts only with one ureylbenzamide unit (structure III) and another structure where both units participate in the complexation (structure IV). This calculation was done in order to elucidate which complex would be favored based on their total energy. The results indicated that structure IV was $9.76 \mathrm{kcal} \mathrm{mol}^{-1}$ more stable than structure III (Fig. 9). As can be seen in Fig. 9a, complex IV showed more hydrogen bond interactions between the receptor and the $\mathrm{AcO}^{-}$ compared with complex III and the nitrophenyl groups appear face to face, indicating a possible additional interaction. This structure agrees with the chemical shifts and the signals affected during the titration with TBAA by NMR, which are very similar to those observed with the mono-ureylbenzamide (Fig. 4).

The complexes with $\mathrm{H}_{2} \mathrm{PO}_{4}{ }^{-}$and $\mathrm{HP}_{2} \mathrm{O}_{7}{ }^{3-}$ were also optimized (Fig. 10). In the $1: 1 \mathbf{4} \mathbf{b}-\mathrm{H}_{2} \mathrm{PO}_{4}{ }^{-}$complex (structure $\mathbf{V}$ ), both urea units interacted with the anion through the $\mathrm{N}-\mathrm{H}_{2}$, leaving the $\mathrm{N}-\mathrm{H}_{1}$ free, while only one of the $\mathrm{N}-\mathrm{H}_{3}$ interacted with the anion. This means that three sites were available to interact with a second anion. In fact, the NMR experiment showed a wide signal for $\mathrm{H}_{1}$ with the TBAHP addition but no shift to downfield was observed (see Fig. S32 $\dagger$ ). This result was consistent with the experimental observed tendency of these receptors to form multi-equivalent complexes with $\mathrm{H}_{2} \mathrm{PO}_{4}{ }^{-}$. The complex with $\mathrm{HP}_{2} \mathrm{O}_{7}{ }^{3-}$ showed deprotonation of the $\mathrm{N}-\mathrm{H}_{2}$ in one urea unit. This acid-base reaction was evident in the NMR titrations, and a change of color in the solution was also observed. Almost all the hydrogen bonding sites interacted with the anion in the complex.

It was noteworthy that the complexes of $\mathbf{4 c}$ and $\mathbf{4 d}$ with $\mathrm{AcO}^{-}$ showed a helical conformation, where both ureylbenzamide units had a coplanar and antiparallel position allowing the interaction with the anion through five hydrogen bonds (Fig. 11). The enhancement of the spacer chain from four to six methylenes favored the coplanarity between the two units. The complex with $\mathrm{BnO}^{-}$showed a similar helical conformation (Fig. S64 $\dagger$ ). This singular conformation with the carboxylate anions may induce the exclusive formation of $1: 1$ complexes, as identified by the UV-vis and NMR techniques. The helical conformation of receptor $\mathbf{4 d}$ was distorted in the complexes with $\mathrm{H}_{2} \mathrm{PO}_{4}{ }^{-}$and $\mathrm{HP}_{2} \mathrm{O}_{7}{ }^{3-}$ due to the differences in the sizes and geometry of these anions (Fig. S64 $\dagger$ ).

The $1: 1$ complex of receptor $\mathbf{8 b}$ with acetate was optimized at the same level of theory. The "saddle" shape of the receptor in the complex allowed the $\mathrm{H}_{1}, \mathrm{H}_{2}, \mathrm{H}_{3}$, and $\mathrm{H}_{4}$ hydrogens of both ureylbenzamide units to be oriented in the direction of the anion (Fig. 12a and b). The distance between the anion and the interacting groups were minimal and the hydrogen bonding was established in the cavity of the receptor in this arrangement. The ureylbenzamide units adopted a perpendicular position to form a compact cavity, where $\mathrm{H}_{1}, \mathrm{H}_{2}, \mathrm{H}_{3}$, and $\mathrm{H}_{4}$ were oriented to the anion in the complex of $\mathbf{8 d}$ with acetate (Fig. 12c and d). The flexibility of the oligomethylene spacer chain allowed the necessary conformational changes to form a 1:1 complex despite the distance between both ureylbenzamide in these receptors (Fig. S65 $\dagger$ ).

The optimization of the complexes with phosphate and pyrophosphate showed configurational changes in the oligomethylene chain induced by the size and geometry of the anion in order to minimize the distance between the interaction sites and the anions (Fig. S66 and S67 $\dagger$ ). Interestingly, the complexes with $\mathbf{8 b}$ showed both anions located in a superficial position on the cavity formed by the two ureylbenzamide units. The distances allowed the interaction of the anions with the $\mathrm{H}_{1}, \mathrm{H}_{2}$, $\mathrm{H}_{3}$, and $\mathrm{H}_{4}$ hydrogens. The receptors $8 \mathbf{d}$ and $8 \mathbf{e}$ with a longer oligomethylene spacer formed a big cavity and the anions occupied an internal or equatorial position. These observations partially explain or justify the high values of $\log K$ for $1: 1$ complexes in receptors with long spacer chain.

\section{Conclusions}

The results obtained by UV-vis and ${ }^{1} \mathrm{H}$ NMR as well as the values of $\log K$ and the theoretical analysis revealed a strong interaction of receptors $\mathbf{4}$ and $\mathbf{8}$ with the anions due to their bifunctionality. The flexible oligomethylene chain in these receptors enhance the possibility to adopt different conformational arrangement to form 1:1 complexes regardless of differences in the size and shape of the anion. Receptors 4 formed 1:1 complexes with carboxylates due to the singular helical conformation of the receptors, which is an obvious consequence of the relative ortho position of the urea and amide groups. The anions with different sizes and geometries also formed 1 : 1 complexes, but the helical conformation was distorted to maximize the interactions. These results also revealed the formation of complexes with variable stoichiometry with dihydrogen phosphate and pyrophosphate due to their geometry, charge number, and autoassociation process.

\section{Conflicts of interest}

There are no conflicts to declare.

\section{Acknowledgements}

Authors thank Consejo Nacional de Ciencia y Tecnología (CONACyT Mexico) (Grant No. CB-2014-239581) for financial support for this project. José García-Elías thanks to CONACyT for the graduate fellowship. The authors acknowledge grateful support from the Supramolecular Chemistry Thematic Network (CONACyT Grant No. 294810). Also, thank CONACyT for the ITT NMR facilities (Grant INFR-201103-173395). 


\section{References}

$1 \mathrm{X}$. Ma and Y. Zhao, Biomedical Applications of Supramolecular Systems Based on Host-Guest Interactions, Chem. Rev., 2015, 115, 7794-7839.

2 S. N. Berry, V. Soto-Cerrato, E. N. W. Howe, H. J. Clarke, I. Mistry, A. Tavassoli, Y.-T. Chang, R. Pérez-Tomás and P. A. Gale, Fluorescent Transmembrane Anion Transporters: Shedding Light on Anionophoric Activity in Cells, Chem. Sci., 2016, 7, 5069-5077.

3 P. A. Gale, E. N. W. Howe and X. Wu, Anion Receptor Chemistry, Chem, 2016, 1, 351-422.

4 P. A. Gale, Anion Receptor Chemistry: Highlights from 1999, Coord. Chem. Rev., 2001, 213, 79-128.

5 M. Boiocchi, L. Del Boca, D. E. Gómez, L. Fabbrizzi, M. Licchelli and E. Monzani, Nature of Urea-Fluoride Interaction: Incipient and Definitive Proton Transfer, $J$. Am. Chem. Soc., 2004, 126, 16507-16514.

6 D. A. Jose, D. K. Kumar, P. Kar, S. Verma, A. Ghosh, B. Ganguly, H. N. Ghosh and A. Das, Role of Positional Isomers on Receptor-anion Binding and Evidence for Resonance Energy Transfer, Tetrahedron, 2007, 63, 1200712014.

7 T. H. Russ, A. Pramanik, M. E. Khansari, B. M. Wong and M. A. Hossain, A Quinoline Based Bis-Urea Receptor for Anions: A Selective Receptor for Hydrogen Sulfate, Nat. Prod. Commun., 2012, 7, 301-304.

8 V. Amendola, D. Esteban-Gómez, L. Fabbrizzi and M. Licchelli, What Anions Do to N-H-Containing Receptors, Acc. Chem. Res., 2006, 39, 343-353.

9 C. M. G. dos Santos, T. McCabe, G. W. Watson, P. E. Kruger and T. Gunnlaugsson, The Recognition and Sensing of Anions through "Positive Allosteric Effects" Using Simple Urea-Amide Receptors, J. Org. Chem., 2008, 73, 9235-9244.

$10 \mathrm{P}$. Comba, The Relation between Ligand Structures, Coordination Stereochemistry, and Electronic and Thermodynamic Properties, Coord. Chem. Rev., 1993, 123, 1-48.

11 V. S. Bryantsev and B. P. Hay, De Novo Structure-Based Design of Bisurea Hosts for Tetrahedral Oxoanion Guests, J. Am. Chem. Soc., 2006, 128, 2035-2042.

12 B. P. Hay, T. K. Firman and B. A. Moyer, Structural Design Criteria for Anion Hosts: Strategies for Achieving Anion Shape Recognition through the Complementary Placement of Urea Donor Groups, J. Am. Chem. Soc., 2005, 127, 18101819.

13 B. Garg, T. Bisht and S. M. S. Chauhan, 2,2'-DiaminoazoBenzene, a Potential Scaffold for the Synthesis of Bis-Ureas and Thioureas: Solution Phase Anion Sensing and Binding Studies, Sens. Actuators, B, 2012, 168, 318-328.

14 S. Nishizawa, P. Bühlmann, M. Iwao and Y. Umezawa, Anion Recognition by Urea and Thiourea Groups: Remarkably Simple Neutral Receptors for Dihydrogenphosphate, Tetrahedron Lett., 1995, 36, 6483-6486.
15 P. Bühlmann, S. Nishizawa, K. P. Xiao and Y. Umezawa, Strong Hydrogen Bond-Mediated Complexation of $\mathrm{H}_{2} \mathrm{PO}_{4}{ }^{-}$ by Neutral Bis-Thiourea Hosts, Tetrahedron, 1997, 53, 1647-1654.

16 S. Nishizawa, P. Bühlmann, K. P. Xiao and Y. Umezawa, Application of a Bis-Thiourea Ionophore for an Anion Selective Electrode with a Remarkable Sulfate Selectivity, Anal. Chim. Acta, 1998, 358, 35-44.

17 A. N. Leung, D. A. Degenhardt and P. Bühlmann, Effect of Spacer Geometry on Oxoanion Binding by Bis- and Tetrakis-Thiourea Hosts, Tetrahedron, 2008, 64, 2530-2536.

18 Y.-J. Kim, H. Kwak, S. J. Lee, J. S. Lee, H. J. Kwon, S. H. Nam, K. Lee and C. Kim, Urea/thiourea-Based Colorimetric Chemosensors for the Biologically Important Ions: Efficient and Simple Sensors, Tetrahedron, 2006, 62, 96359640.

19 C. Caltagirone, C. Bazzicalupi, F. Isaia, M. E. Light, V. Lippolis, R. Montis, S. Murgia, M. Olivari and G. Picci, A New Family of Bis-Ureidic Receptors for Pyrophosphate Optical Sensing, Org. Biomol. Chem., 2013, 11, 2445-2451.

20 B. Moreno-Valle, M. Aguilar-Martínez, A. Ochoa-Terán, M. Martínez-Quiroz, V. Miranda-Soto, J. García-Elías, K. Ochoa-Lara, V. Labastida-Galván and M. Ordoñez, Synthesis and Anion Recognition Studies of New Ureylbenzamide-Based Receptors, Supramol. Chem., 2018, 30, 9-19.

21 P. Gans, A. Sabatini and A. Vacca, Talanta, 1996, 43, 17391753.

22 P. Gans, A. Sabatini and A. Vacca, Anal. Chim. Acta, 1999, 89, 45-49.

23 A. Shokrollahi, F. Zarghampour, S. Akbari and A. Salehi, Anal. Methods, 2015, 7, 3551-3558.

24 M. J. Frisch, G. W. Trucks, H. B. Schlegel, G. E. Scuseria, M. A. Robb, J. R. Cheeseman, G. Scalmani, V. Barone, B. Mennucci, G. A. Petersson, H. Nakatsuji, M. Caricato, X. Li, H. P. Hratchian, A. F. Izmaylov, J. Bloino, G. Zheng, J. L. Sonnenberg, M. Hada, M. Ehara, K. Toyota, R. Fukuda, J. Hasegawa, M. Ishida, T. Nakajima, Y. Honda, O. Kitao, H. Nakai, T. Vreven, J. A. Montgomery, J. E. Peralta, F. Ogliaro, M. Bearpark, J. J. Heyd, K. N. Brothers V. N. Kudin R. Staroverov J. Kobayashi K. Normand E. Raghavachari, A. Rendell, J. C. Burant, S. S. Iyengar, J. Tomasi, M. Cossi, N. Rega, J. M. Millam, M. Klene, J. E. Knox, J. B. Cross, V. Bakken, C. Adamo, J. Jaramillo, R. Gomperts, R. E. Stratmann, O. Yazyev, A. J. Austin, R. Cammi, C. Pomelli, J. W. Ochterski, R. L. Martin, K. Morokuma, V. G. Zakrzewski, G. A. Voth, P. Salvador, J. J. Dannenberg, S. Dapprich, A. D. Daniels, O. Farkas, J. B. Foresman, J. V. Ortiz, J. Cioslowski and D. J. Fox, Gaussian 09, Revision E.01, Gaussian, Inc., Wallingford CT, 2013.

25 D. Barisic, V. Tomisic and N. Bregovic, Anal. Chim. Acta, 2019, 1046, 77-92. 